\title{
Broadband spectral analysis of MXB 1659-298 in its soft and hard state
}

\author{
R. Iaria ${ }^{1}$, S. M. Mazzola ${ }^{1}$, T. Bassi ${ }^{1,2,3}$, A. F. Gambino ${ }^{1}$, A. Marino ${ }^{1,2,3}$, T. Di Salvo ${ }^{1}$, A. Sanna ${ }^{4}$, A. Riggio ${ }^{4}$, \\ L. Burderi ${ }^{4}$, and N. D'Amico ${ }^{4,5}$ \\ 1 Università degli Studi di Palermo, Dipartimento di Fisica e Chimica, Via Archirafi 36, 90123 Palermo, Italy \\ e-mail: rosario.iaria@unipa.it \\ 2 INAF - Istituto di Astrofisica Spaziale e Fisica Cosmica di Palermo, Via Ugo La Malfa 153, 90146 Palermo, Italy \\ 3 IRAP, Université de Toulouse, CNRS, UPS, CNES, Toulouse, France \\ 4 Università degli Studi di Cagliari, Dipartimento di Fisica, SP Monserrato-Sestu, KM 0.7, 09042 Monserrato, Italy \\ 5 INAF, Osservatorio Astronomico di Cagliari, Via della Scienza 5, 09047 Selargius, CA, Italy
}

Received 29 July 2018 / Accepted 5 September 2019

\begin{abstract}
Context. The X-ray transient eclipsing source MXB 1659-298 went into outburst in 1999 and 2015. During these two outbursts the source was observed by XMM-Newton, NuSTAR, and Swift/XRT.

Aims. Using these observations, we studied the broadband spectrum of the source to constrain the continuum components and to verify whether it had a reflection component, as is observed in other X-ray eclipsing transient sources.

Methods. We combined the available spectra to study the soft and hard state of the source in the $0.45-55 \mathrm{keV}$ energy range

Results. We report a reflection component in the soft and hard state. The direct emission in the soft state can be modeled with a thermal component originating from the inner accretion disk plus a Comptonized component associated with an optically thick corona surrounding the neutron star. On the other hand, the direct emission in the hard state is described only by a Comptonized component with a temperature higher than $130 \mathrm{keV}$; this component is associated with an optically thin corona. We observed narrow absorption lines from highly ionized ions of oxygen, neon, and iron in the soft spectral state. We investigated where the narrow absorption lines form in the ionized absorber. The equivalent hydrogen column density associated with the absorber is close to $6 \times 10^{23} \mathrm{~cm}^{-2}$ and $1.3 \times 10^{23} \mathrm{~cm}^{-2}$ in the soft and hard state, respectively.
\end{abstract}

Key words. stars: neutron - stars: individual: MXB 1659-298 - X-rays: binaries - accretion, accretion disks

\section{Introduction}

Low-mass X-ray binaries hosting neutron stars (hereafter NS-LMXBs) are binary systems in which a weakly magnetized neutron star (NS) accretes matter from a low-mass $\left(<1 M_{\odot}\right)$ companion star through Roche-lobe overflow. The NS-LMXBs display several spectral states and hysteresis patterns similar to that observed in X-ray binary systems harboring a black hole (BH) as compact object, as shown by Muñoz-Darias et al. (2014). The hysteresis pattern highlights the soft, hard, and intermediate states. This suggests a similarity between these two classes of X-ray sources. The NS-LMXB spectrum in the soft state shows a dominant soft emission associated with a blackbody or a disk-blackbody component with a temperature between 0.5 and $2 \mathrm{keV}$ and a harder $(3-5 \mathrm{keV})$ saturated Comptonized component with a high optical depth. The thermal component originates from the NS surface and/or from the innermost region of the accretion disk, while the Comptonized component is associated with inverse Compton emission that is due to the interaction of the soft photons emitted from the innermost region with the electrons in the thermal corona surrounding the NS (see, e.g., di Salvo et al. 2009; Iaria et al. 2009, and references therein). On the other hand, the hard-state spectrum can be described by a weak (or absent) thermal component plus a cutoff power-law component, with a cutoff energy higher than $10 \mathrm{keV}$ that is produced by inverse Compton scattering of soft photons in a hot optically thin electron corona (see, e.g., Di Salvo et al. 2015; Cackett et al. 2010, and references therein).

Studying the reflection component that originates from direct Compton scattering of the photons that are emitted by the hot corona with the cold electrons in the top layers of the inner accretion disk is of particular interest. A peculiar feature in the continuum emission is the Compton hump around $20-40 \mathrm{keV}$. It can be ascribed to direct Compton scattering and is observed mainly when the source is in the hard state because the flux above $20 \mathrm{keV}$ is higher than in soft state (see, e.g., Egron et al. 2013; Miller et al. 2013, and references therein). The reflection component also shows broad emission lines (FWHM close to $1 \mathrm{keV}$ ) in the Fe-K region, which are often observed in the spectra of NS-LMXBs with an inclination angle lower than $60^{\circ}$ (see, e.g., Iaria et al. 2016, 2009; Pandel et al. 2008; Shaposhnikov et al. 2009) and inclination angles between $60^{\circ}$ and $80^{\circ}$ (the so-called dipping and eclipsing sources, e.g., Iaria et al. 2007; Ponti et al. 2015). These lines are identified with the $\mathrm{K} \alpha$ transitions of iron at different ionization states. Compton scattering is not sufficient to explain the large width measured for these lines (Sanna et al. 2013; Reis et al. 2009). A possible scenario that allows explaining the large observed broadening is that these lines originate in the innermost region of the accretion disk, close to the compact object, where matter reaches Keplerian velocities up to a few tenths of the speed of light. For this reason the reflection component is corrected by relativistic Doppler effects and gravitational redshift (Fabian et al. 1989). 
MXB 1659-298 is an eclipsing transient source that shows type I X-ray bursts. Iaria et al. (2018) and Jain et al. (2017), studying the eclipse arrival times of the source obtained during the outburst that occurred between 1999 and 2001 and the outburst between 2015 and 2017, suggested that a third body might orbit the binary system. Iaria et al. (2018) inferred an orbital period of the binary of 7.1161099(3) $\mathrm{hr}$ and an orbital period derivative of $-8.5(1.2) \times 10^{-12} \mathrm{~s} \mathrm{~s}^{-1}$; they also inferred an inclination angle of $72^{\circ} \pm 3^{\circ}$. The distance to the source was estimated to be $9 \pm 2$ and $12 \pm 3 \mathrm{kpc}$ for a hydrogen-rich and helium-rich companion star, respectively (Galloway et al. 2008).

Studying the XMM-Newton spectrum of MXB 1659-298, Sidoli et al. (2001) detected two absorption lines at 6.64 and $6.90 \mathrm{keV}$ associated with Fe XXV and Fe XXVI ions, as well as absorption lines associated with the transitions of $\mathrm{O}$ VIII at 0.65 , $0.77,0.81 \mathrm{keV}$ and $\mathrm{Ne}$ IX at $1.0 \mathrm{keV}$, respectively. Furthermore, the authors detected a broad emission line centered at $6.47 \mathrm{keV}$ with a FWHM of $1.4 \mathrm{keV}$ that can be associated with a K-shell transition of neutral or weakly ionized iron.

Recently, Sharma et al. (2018) analyzed the 0.5-30 keV soft spectral state of the source using Swift/XRT and NuSTAR data and fit the continuum emission with a model composed of a thermal component and a Comptonized component with seed photons emitted from the NS surface. The same authors analyzed the hard spectral state using Swift/XRT and NUSTAR spectra in the $0.5-70 \mathrm{keV}$ energy band and adopting a model composed of two thermal components produced at the NS surface and at the innermost region of the disk plus a Comptonized component in which the seed photons are emitted from the NS surface. The same authors also investigated an ionized absorber in the soft and hard spectral states, finding that this is present in the soft state with an ionization parameter $\log (\xi)$ close to 4 and a corresponding equivalent column of neutral hydrogen of $7 \times 10^{23} \mathrm{~cm}^{-2}$, while the ionized absorber is not statistically significant in the hard spectral state.

Ponti et al. (2019) used Chandra/HETG and NuSTAR data to analyze the $0.8-30 \mathrm{keV}$ soft spectral state and XMM-Newton data to analyze the $0.5-10 \mathrm{keV}$ hard spectral state of MXB 1659-298. The HETG spectrum shows the presence a several absorption lines of $\mathrm{H}$-like and He-like ions of $\mathrm{Ne}, \mathrm{Mg}, \mathrm{Si}, \mathrm{S}, \mathrm{Ar}, \mathrm{Ca}, \mathrm{Fe}$, and $\mathrm{Ni}$. The adopted continuum emission is composed of two thermal components corresponding to the emission from the NS surface and the innermost region of the accretion disk plus a Comptonized component associated with the corona surrounding the NS. The authors also suggest the presence of a broad emission line in the Fe-K region of the spectrum that was interpreted as a blurred relativistic line. The absorption lines were ascribed to an ionized absorber that has a ionization parameter $\log (\xi)$ close to 4.5 and a corresponding equivalent column of neutral hydrogen of $2.4 \times 10^{23} \mathrm{~cm}^{-2}$.

The behavior of MXB 1659-298 is similar to that of the eclipsing transient source AX J1745.6-2901. A broadband study of AX J1745.6-2901 was performed by Ponti et al. (2015), who analyzed the source during its outbursts using XMM-Newton, NuSTAR, and Swift/XRT data. The authors studied the soft and hard spectral states and found that the persistent emission is absorbed by a column density of neutral material of $N_{\mathrm{H}} \simeq 1.9 \times$ $10^{23} \mathrm{~cm}^{-2}$ and concluded that most of the (photoelectric) obscuring column density is due to the interstellar medium. Moreover, the same authors detected Fe K absorption lines in the soft state that disappeared during the hard state. The column densities and turbulent velocities of the absorbing ionized plasma are higher than $10^{23} \mathrm{~cm}^{-2}$ and $500 \mathrm{~km} \mathrm{~s}^{-1}$, respectively. Finally, they found that the continuum emission in the soft state requires a relativis- tic line component with an equivalent width between 80 and $200 \mathrm{eV}$.

In this paper we report the broadband spectral analysis of the persistent X-ray emission of MXB 1659-298 during the 1999 and 2015 outbursts using XMM-Newton, NuSTAR, and Swift/XRT data. We analyzed the X-ray spectra in the soft and hard state and found that a relativistic reflection component is necessary to fit the spectra; furthermore, we observed absorption lines associated with highly ionized iron that we modeled using an ionized absorber. We discuss the nature of the ionized absorber in the soft spectral state where the absorption lines are more prominent.

\section{Observation and data reduction}

\subsection{XMM-Newton observations}

The XMM-Newton Observatory (Jansen et al. 2001) observed MXB 1659-298 twice. The first observation (ObsId. 0008610701) was performed on 2001 February 20 between 8:28:27 UTC and 16:2:39 UTC for $31.5 \mathrm{ks}$, during the outburst that occurred from April 1999 until September 2001. The source was observed a second time for $42.9 \mathrm{ks}$ between 2015 September 26 19:53:05 UTC and September 27 06:53:47 UTC, during its last outburst (Obsid. 0748391601). The first and second XMM-Newton observation have been studied by Sidoli et al. (2001) and Ponti et al. (2019), respectively.

The PN-type CCD detector of the European Photon Imaging Camera (EPN; Strüder et al. 2001) was operating in Small Window Imaging (SW) mode during the first observation and in Timing mode during the second observation. The Reflecting Grating Spectrometer (RGS, two modules, den Herder et al. 2001) was operating in standard spectroscopy mode during these two observations. We reprocessed the XMM-Newton data using the Science Analysis Software (SAS) v16.1.0, obtaining the calibrated photon event files using the SAS tools epproc and rgsproc. We verified the absence of background flaring in the EPN data by extracting the light curve in the $10-12 \mathrm{keV}$ energy range for all the observations. The EPN events were selected between 0.3 and $12 \mathrm{keV}$ using only single and double pixel events $($ PATTERN $\leq 4)$ that were optimally calibrated for spectral analysis $(F L A G=0)$. For our aim, we considered only the RGS and EPN events during the persistent emission, excluding dips, eclipses, and type I X-ray bursts. The RGS spectra (hereafter RGS12 spectra) were obtained by combining the first-order spectra of RGS1 and RGS2 using the SAS tool rgscombine after verifying that the two spectra were similar to each other.

The EPN light curve of the persistent emission corresponding to the observation taken in 2001 was obtained using a circular region with a radius of $50^{\prime \prime}$. We found a count rate higher than 140 counts s$^{-1}$. Because pile-up issues are expected to be significant at count rates higher than 25 counts s $^{-1}$ in EPN observations performed in SW mode, we extracted the source and background events using an annular region with inner and outer radius of $8.1^{\prime \prime}$ and $50^{\prime \prime}$, respectively, to mitigate the pile-up issues. We verified the goodness of our choice using the SAS tool epatplot. The $0.3-12 \mathrm{keV}$ EPN background-subtracted light curve is shown in the left panel of Fig. 1. The light curve shows two dips, two total eclipses, and a type I X-ray burst. The count rate during the persistent emission is between 70 and 80 counts s$^{-1}$ and the corresponding exposure time is $12.8 \mathrm{ks}$. The RGS12 spectrum has an exposure time of $34.6 \mathrm{ks}$.

The source events of the second observation were extracted from a box region that included the brightest columns of the 

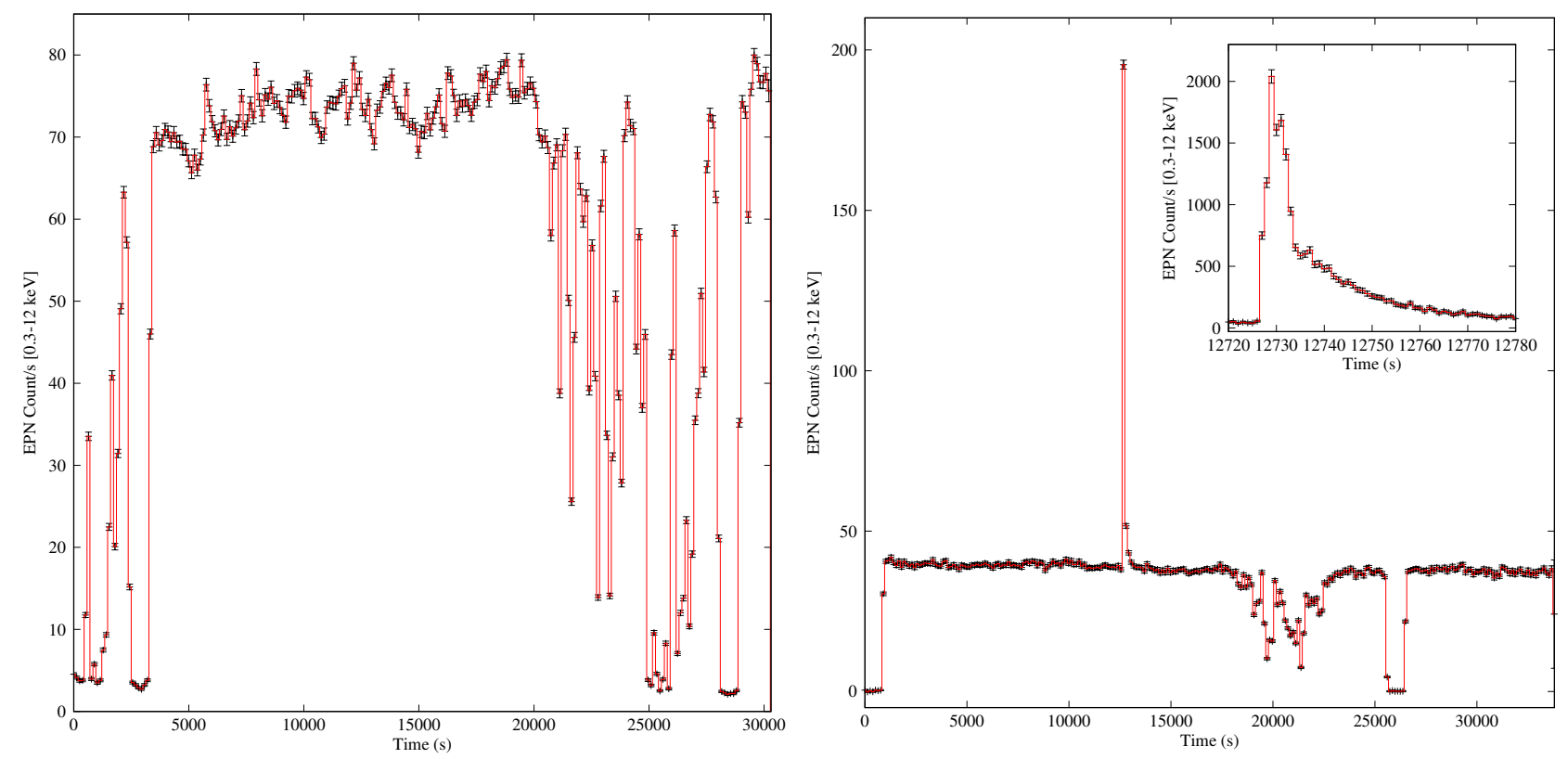

Fig. 1. EPN background-subtracted light curve in the $0.3-12 \mathrm{keV}$ energy range corresponding to the XMM-Newton observations taken in 2001 (left panel) and 2015 (right panel). The bin time is $128 \mathrm{~s}$. Inset: shape of the type I X-ray burst with a binning time of $1 \mathrm{~s}$.

detector and had a width of $127^{\prime \prime}$ (RAWX between 22 and 52). The background events were extracted from a box region far away from the source with a width of 10" (RAWX between 5 and 10). We show the EPN background-subtracted light curve in the right panel of Fig. 1. The light curve shows a dip at $20 \mathrm{ks}$ from the start time, two eclipses (the first at the start time of the observation and the second $26 \mathrm{ks}$ after the start time) and a type I X-ray burst at $12.7 \mathrm{ks}$. We excluded the events collected later $33.7 \mathrm{ks}$ from the beginning of the observation because they were affected by an instrument detection failure. The count rate during the persistent emission is 40 counts $^{-1}$; because the EPN operated in Timing Mode, we can exclude pile-up issues for this observation. The RGS12 and EPN spectra have an exposure time of 68.7 and $26 \mathrm{ks}$, respectively. We adopted energy ranges of $0.4-2 \mathrm{keV}$ for the RGS12 spectra and $0.6-12 \mathrm{keV}$ for the EPN spectra.

\subsection{NUSTAR observations}

During the outburst that occurred between 2015 and 2017, MXB 1659-298 was observed twice by the Nuclear Spectroscopic Telescope Array satellite (NuSTAR; Harrison et al. 2013): the first observation (ObsId. 90101013002) was performed between 2015 September 28 21:51:08 UTC and September 30 00:56:08 UTC for an exposure time of $51.5 \mathrm{ks}$, the second observation (ObsId. 90201017002) was carried out between 2016 April 21 14:41:08 UTC and April 22 04:56:08 UTC for an exposure time of $26.8 \mathrm{ks}$. The analysis of the observation taken in 2015 was also reported by Sharma et al. (2018), and the observation taken in 2016 was reported by Sharma et al. (2018) and Ponti et al. (2019).

The data were processed using the NUSTAR Data Analysis Software (NuSTAR-DAS) v1.9.3 for the data sets taken by the focal plane modules, FPMA and FPMB, and for both observations. The source events were extracted from a circular region centered on the source coordinates that had a radius of $110^{\prime \prime}$. The filtered events, the background-subtracted light curves, the spectra, and the arf and rmf files were created using nuproducts tool.
We show the 1.6-80 keV FPMA background-subtracted light curves for the first and second NUSTAR observation in Fig. 2. We identified two dips, two eclipses, and one type I X-ray burst in the light curve corresponding to the first observation, while in the second observation we observed seven type I X-ray bursts, one eclipse, one almost complete dip at $25 \mathrm{ks}$ after the start time, and part of two dips at the beginning and end of the observation. The count rate during the persistent emission is close to 4 count s ${ }^{-1}$ and 20 count $\mathrm{s}^{-1}$ during the first and second observation, respectively.

To produce the FPMA and FPMB spectra associated with the persistent emission, we created, using XSELECT v2.4d, the good time intervals (gti) files to exclude the type I X-ray bursts, the eclipses, and the dips. Finally, after verifying the good agreement between the FPMA and FPMB spectra, we used the addascaspec task to obtain a single combined spectrum. The exposure times of the combined NUSTAR persistent spectrum are $92.8 \mathrm{ks}$ and $44.3 \mathrm{ks}$ for the first and the second observation, respectively. We adopted energy ranges of $3-35 \mathrm{keV}$ and $3-55 \mathrm{keV}$ for the spectra extracted from the observations taken in 2015 and 2016, respectively.

\subsection{Swift/XRT observation}

MXB 1659-298 was monitored by the X-Ray Telescope (XRT; Burrows et al. 2005) on board the Swift Observatory (Gehrels et al. 2004). We analyzed the Swift/XRT observations 00034002036 and 00081918001 taken on 2016 April 20 01:47:54 and 2016 April 21 20:39:01, respectively, that is, $37 \mathrm{~h}$ before and $7 \mathrm{~h}$ after the beginning of the NUSTAR observation taken in 2016. The two observations lasted for $803 \mathrm{~s}$ and $697 \mathrm{~s}$, respectively, and were performed in Window Timing (WT) mode $^{1}$. We used the software package HEASOFT (v.6.20) with the Swift Calibration Database (CALDB v.20160609), running the task xrtpipeline.

During the first observation, the roll angle of the satellite (for more details see Burrows et al. 2005) changed, which pro-

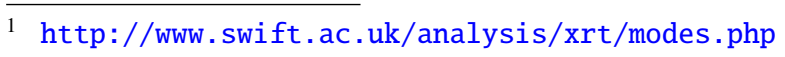



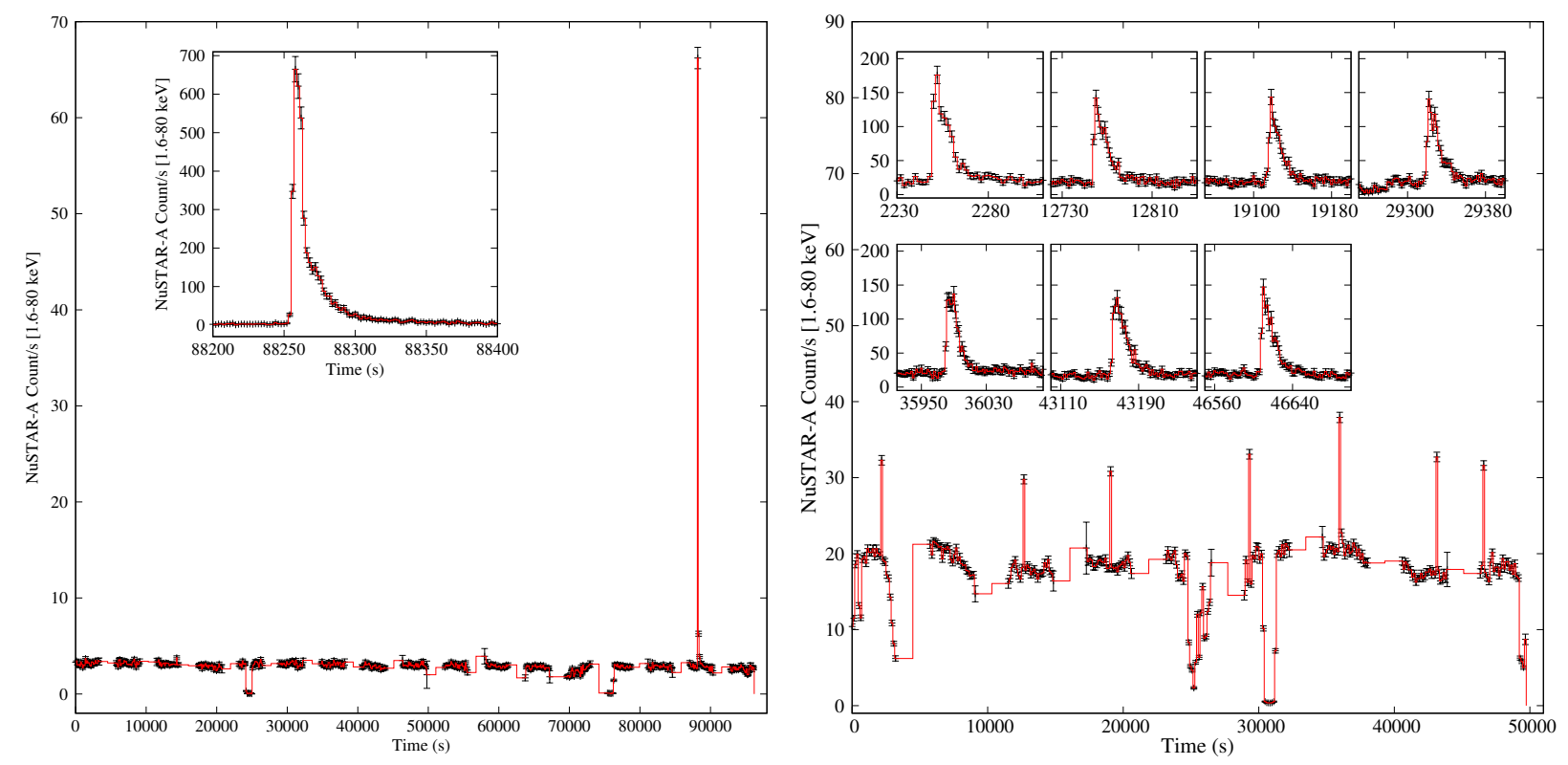

Fig. 2. FPMA light curves obtained from the observations taken in 2015 (left) and in 2016 (right). The bin time is $128 \mathrm{~s}$. The details of the observed $\mathrm{X}$-ray type I bursts are shown in the inset panels with a bin time of $1 \mathrm{~s}$.

duced two different strips in the image of the source. For this reason we generated two separate event files with the ftool XSELECT (v. 2.4d), one for each strip, by extracting the corresponding products. We extracted the source spectra from a circular region with a radius of 20 pixel $\left(1\right.$ pixel $\left.=2.357^{\prime \prime}\right)$ centered on the source position using the task xrtproducts. The background was extracted from a same-size region that was free from the source. The two spectra have exposure times of $669 \mathrm{~s}$ and $134 \mathrm{~s}$, respectively. The second observation was not affected by the change in roll angle, and we extracted the source and background spectra using the same criteria described above. We verified that the spectral shape was compatible among the three spectra and summed them using the FTOOLS addascaspec. The combined spectrum has an exposure time of $1.4 \mathrm{ks}$ and it was rebinned to have at least 20 counts per energy bin so that we could apply the $\chi^{2}$ statistics. The adopted spectral energy range is $0.5-9 \mathrm{keV}$.

\section{Spectral analysis}

We combined the fits of the spectra obtained from the XMMNewton and NUSTAR observations taken in 2015 because they show a similar flux of $5 \mathrm{mCrab}$, while the spectra obtained from the XMM-Newton observation taken in 2001 and the NuSTAR and Swift/XRT observations taken in 2016 were fit together because they show a flux close to $30 \mathrm{mCrab}$ (see the ASM and MAXI/GSC light curves, shown in Fig. 1 by Iaria et al. 2018). Hereafter we call the two combined spectra low- and high-flux spectrum, respectively.

We adopted the cosmic abundances and the cross sections derived by Wilms et al. (2000) and Verner et al. (1996a), respectively. With the aim to account for the interstellar absorption, we adopted the Tübingen-Boulder model (TBABS in XSPEC). The uncertainties are reported at $90 \%$ confidence level (c.l.).

\subsection{High-flux spectrum (the soft state)}

We adopted a model that takes into account an emission from the accretion disk (DISKBB in XSPEC, see Mitsuda et al. 1984) plus a thermally Comptonized component (NTHCOMP in XSPEC, see
Życki et al. 1999) to fit the continuum emission. Because the RGS12 and EPN events were collected at different times than the Swift/XRT and NuSTAR events (i.e., 2001 and 2016, respectively), we left the seed photon temperature $k T_{\mathrm{bb}}$, the electron temperature $k T_{\mathrm{e}}$, and the asymptotic power-law photon index $\Gamma$ free to vary independently. Moreover, we fixed the parameter INP_TYPE of NTHCOMP to zero, assuming that the seed photons have a blackbody spectrum (i.e., the seed photons originate from the NS surface). Because large residuals were evident at $0.54 \mathrm{keV}$ in the RGS12 spectrum, we added an absorption edge only to the RGS12 spectrum. This feature is interpreted as a calibration issue associated with the $\mathrm{K}$ edge of neutral oxygen in the RGS spectra (de Vries et al. 2003). We modeled the Au instrumental edge $(\sim 2.2 \mathrm{keV})$ in the EPN spectrum adding a Gaussian component, as was done by Pintore et al. (2016) and Sanna et al. (2017). We fixed the energy of the line at $2.22 \mathrm{keV}$ and imposed a null width. The Gaussian normalization was left free to vary for the EPN spectrum but was fixed to zero for the other spectra. Then the initial adopted model was

Model $1=$ Edge $*$ TBabs $*($ diskbb + nthComp $)$.

We found a $\chi^{2}$ (d.o.f.) of 4121(2801). We show the best-fit parameters of Model 1 in the third column of Table 1 . The residuals are shown in the second panel from the top in Fig. 3. We observed that large residuals were present between 6 and $10 \mathrm{keV}$. These can be interpreted as absorption lines and edges associated with the presence of Fe XXV and Fe XXVI ions. Furthermore, the RGS12 spectrum showed two absorption lines at 0.6 and $1.02 \mathrm{keV}$ that might be associated with $\mathrm{O}$ VIII and $\mathrm{Ne} \mathrm{X}$ ions.

To fit these absorption features, we added a multiplicative component, ZXIPCF $^{2}$, to Model 1 that takes into account a partial covering of ionized absorbing material. This component reproduces the absorption from photoionized matter that is illuminated by a power-law source with spectral index $\Gamma=2.2$ and assumes that the photoionized absorber has a microturbulent velocity of $200 \mathrm{~km} \mathrm{~s}^{-1}$. The component ZXIPCF

\footnotetext{
2 https://heasarc.gsfc.nasa.gov/xanadu/xspec/models/ zxipcf.html
} 
Table 1. Best-fit parameters of the high-flux spectrum.

\begin{tabular}{|c|c|c|c|c|c|c|c|c|c|}
\hline \multirow[t]{2}{*}{ Model } & \multirow[t]{2}{*}{ Component } & \multicolumn{2}{|c|}{ Model 1} & \multicolumn{2}{|c|}{ Model 2} & \multicolumn{2}{|c|}{$\begin{array}{c}\text { Model } 2 \\
+ \text { Broad Line } \\
\end{array}$} & \multicolumn{2}{|c|}{ Model 3} \\
\hline & & Obs. 2001 & Obs. 2016 & Obs. 2001 & Obs. 2016 & Obs. 2001 & Obs. 2016 & Obs. 2001 & Obs. 2016 \\
\hline \multirow[t]{2}{*}{ EDGE } & $E(\mathrm{keV})$ & \multicolumn{2}{|c|}{$0.529_{-0.010}^{+0.012}$} & \multicolumn{2}{|c|}{$0.536_{-0.003}^{+0.033}$} & \multicolumn{2}{|c|}{$0.537_{-0.004}^{+0.028}$} & \multicolumn{2}{|c|}{$0.541_{-0.011}^{+0.026}$} \\
\hline & $\tau$ & \multicolumn{2}{|c|}{$0.10 \pm 0.03$} & \multicolumn{2}{|c|}{$0.06 \pm 0.03$} & \multicolumn{2}{|c|}{$0.06 \pm 0.02$} & \multicolumn{2}{|c|}{$0.06 \pm 0.02$} \\
\hline \multirow[t]{3}{*}{ ZXIPCF } & $N_{\mathrm{H}}\left(10^{22}\right.$ atoms $\left.\mathrm{cm}^{-2}\right)$ & \multicolumn{2}{|c|}{-} & \multicolumn{2}{|c|}{$120 \pm 20$} & \multicolumn{2}{|c|}{$62 \pm 12$} & \multirow{2}{*}{\multicolumn{2}{|c|}{$\begin{array}{c}60_{-14}^{+9} \\
1 \text { (frozen) }\end{array}$}} \\
\hline & $f_{\mathrm{IA}}$ & \multirow{2}{*}{\multicolumn{2}{|c|}{ - }} & \multicolumn{2}{|c|}{$0.88 \pm 0.07$} & \multicolumn{2}{|c|}{$>0.97$} & & \\
\hline & $\log (\xi)_{\mathrm{IA}}$ & & & $4.45_{-009}^{+0.14}$ & $4.24 \pm 0.05$ & $4.366_{-0.008}^{+0.048}$ & $4.11 \pm 0.03$ & $4.37 \pm 0.04$ & $4.14 \pm 0.05$ \\
\hline TBABS & $N_{\mathrm{H}_{\mathrm{ISM}}}\left(10^{22}\right.$ atoms $\left.\mathrm{cm}^{-2}\right)$ & \multicolumn{2}{|c|}{$0.267 \pm 0.007$} & \multicolumn{2}{|c|}{$0.282_{-0.013}^{+0.008}$} & \multicolumn{2}{|c|}{$0.281 \pm 0.003$} & \multicolumn{2}{|c|}{$0.275 \pm 0.010$} \\
\hline PARTCOV & $f$ & \multicolumn{2}{|c|}{-} & \multirow{2}{*}{\multicolumn{2}{|c|}{$0.88 \pm 0.07$}} & & & & zen) \\
\hline CABS & $N_{\mathrm{H}}\left(10^{22}\right.$ atoms $\left.\mathrm{cm}^{-2}\right)$ & & & & & & & & \\
\hline DISKBB & $k T_{\text {in }}(\mathrm{keV})$ & & +0.009 & & 0.028 & 0.31 & .011 & & 0.02 \\
\hline & $R_{\text {disk }} \sqrt{\cos \theta}(\mathrm{km})$ & & $\begin{array}{l}x_{-1.1}^{+1.8} \\
+1.8\end{array}$ & & & & & & \pm 4 \\
\hline GAUSS & $E(\mathrm{keV})$ & & -1 & & & & .10 & & \\
\hline & $\sigma(\mathrm{keV})$ & & & & & & & & \\
\hline & Norm. $\left(10^{-3} \mathrm{ph} \mathrm{cm}^{-2} \mathrm{~s}^{-1}\right)$ & & & & & & .10 & & \\
\hline RDBLUR & Betor10 & & & & & & & & 1.4 \\
\hline & $R_{\text {in }}\left(G M / c^{2}\right)$ & & & & & & & & \\
\hline & $R_{\text {out }}\left(G M / c^{2}\right)$ & & & & & & & 280 & rozen) \\
\hline & $\theta(\mathrm{deg})$ & & & & & & & & ozen) \\
\hline RFXCONV & $\mathrm{rel}_{\mathrm{refl}}$ & & & & & & & & -0.05 \\
\hline & $\mathrm{Fe}_{\text {abund }}$ & & - & & & & & & zen) \\
\hline & $\cos \theta$ & & & & & & & 0.30 & (rozen) \\
\hline & $\log (\xi)$ & & & & & & & & 0 \\
\hline NTHCOMP & $\Gamma$ & $1.66 \pm 0.03$ & $2.33 \pm 0.03$ & $1.70 \pm 0.02$ & $2.123_{-0.013}^{+0.026}$ & $1.71 \pm 0.05$ & $2.155 \pm 0.005$ & $1.69 \pm 0.02$ & $2.152 \pm 0.015$ \\
\hline & $k T_{\mathrm{e}}(\mathrm{keV})$ & $1.92 \pm 0.04$ & $4.33 \pm 0.10$ & $2.04 \pm 0.04$ & $3.56 \pm 0.05$ & $2.022 \pm 0.012$ & $3.69 \pm 0.02$ & $2.00 \pm 0.03$ & $3.66 \pm 0.04$ \\
\hline & $k T_{\mathrm{bb}}(\mathrm{keV})$ & $0.55 \pm 0.03$ & $0.721 \pm 0.015$ & $0.44 \pm 0.03$ & $0.578_{-0.027}^{+0.027}$ & $0.446_{-0.020}^{+0.020}$ & $0.577_{-0.012}^{+0.012}$ & $0.434_{-0.024}^{+0.02}$ & $0.573_{-0.015}^{+0.015}$ \\
\hline & Norm & 0.063 & $=0.004$ & 0.20 & 0.03 & 0.14 & .012 & 0.14 & 0.012 \\
\hline & $\chi^{2} /$ d.o.f. & & 2801 & & 797 & & & & 2794 \\
\hline
\end{tabular}

Notes. The spectra associated with the 2001 observation are the RGS12 and EPN spectra while the spectra associated with the 2016 observation are the Swift/XRT and NuSTAR ones.

was initially developed to study absorption features in the Fe-K region in AGN (see Reeves et al. 2008) and to study the complex spectra of narrow-line Seyfert 1 galaxies (see Miller et al. 2007). Recently, this component was adopted to fit the absorbing features in the $\mathrm{Fe}-\mathrm{K}$ region that is associated with highly ionized matter surrounding the eclipsing NS-LMXB AX J1745.6-2901 (Ponti et al. 2015) and the dipping source XB 1916-053 (Gambino et al. 2019). The parameters of this component are $N_{\mathrm{H}}, \log (\xi)_{\mathrm{IA}}, f_{\mathrm{IA}}$, and $z . N_{\mathrm{H}}$ describes the equivalent hydrogen column density associated with the ionized absorber, and $\log (\xi)_{\text {IA }}$ describes the ionization degree of the absorbing material. It is defined as $\xi_{\mathrm{IA}}=L_{\mathrm{X}} /\left(n_{\mathrm{H}} r^{2}\right)$, where $L_{\mathrm{X}}$ is the $\mathrm{X}$-ray luminosity incident on the absorbing material, $r$ is the distance of the absorber from the X-ray source, and $n_{\mathrm{H}}$ is the hydrogen atom density of the absorber. $f_{\mathrm{IA}}$ indicates the fraction of the source covered by the absorber, and $z$ gives the redshift of the source and was fixed to zero.

To take into account that the ionized absorber can scatter the radiation out of the line of sight via Thomson or Compton scattering, we added the multiplicative component CABS to the model. The only parameter of the component CABS is $N_{\mathrm{H}}$, which describes the equivalent hydrogen column density associated with the scattering cloud. We tied the value of this parameter to that of the equivalent hydrogen column density associated with the absorbing ionized cloud.

In addition, in order to take into account that the absorbing material might cover only a fraction $f$ of the source, we multiplied the component CABS by the component PARTCOV. The latter is a convolution model that allows converting an absorption component into a partially covering absorption component. The parameter of PARTCOV is $f$, that is, the fraction of the source covered by the absorbing material; we tied its value to that of the parameter $f$ of the component ZXIPCF. Furthermore, we left the ionization parameter of the component ZXIPCF for the spectra taken in 2001 and for those taken in 2016 free to vary independently. In summary, Model 2 can be described as

$$
\begin{array}{r}
\text { Model } 2=\text { Edge } * \text { TBabs } *(\text { partcov } * \text { cabs }) \\
* \text { zxipcf } *(\text { diskbb }+ \text { nthComp }) .
\end{array}
$$

By fitting the spectrum, we obtained a $\chi^{2}$ (d.o.f.) of 3331(2797) and decreases of 790. The addition of the ZXIPCF component is statistically significant, as witnessed by the extremely low outcome of the $F$-test probability of chance improvement with respect to Model 1 , that is, $1.5 \times 10^{-127}$. The residuals associated with the absorption lines at 0.65 and $1.02 \mathrm{keV}$ disappeared, while those in the $6-10 \mathrm{keV}$ energy range were largely reduced. We show the best-fit values of the parameters in the fourth column of Table 1, and the residuals are shown in the third panel from the top in Fig. 3. The equivalent hydrogen column associated with the ionized absorbing material is $(1.2 \pm 0.2) \times 10^{24} \mathrm{~cm}^{-2}$, which is a factor 400 larger than the equivalent hydrogen column associated with the neutral interstellar matter of $\left(2.82_{-0.13}^{+0.08}\right) \times 10^{21} \mathrm{~cm}^{-2}$. The covering fraction of the absorber is $0.88 \pm 0.07$, suggesting that a large part of the source is occulted by the absorber. The ionization parameter $\log (\xi)_{\mathrm{IA}}$ is $4.45_{-0.09}^{+0.14}$ and $4.24 \pm 0.05 \mathrm{erg} \mathrm{cm} \mathrm{s}^{-1}$ for the 2001 and 2016 observation, respectively.

Residuals were still present between 6 and $9 \mathrm{keV}$. These might be associated with a reflection component from the accretion disk. Sidoli et al. (2001) detected a broad emission line in the $\mathrm{Fe}-\mathrm{K}$ region that might be associated with a fluorescence iron line originating from disk reflection. In order to verify the presence of a broad emission line, we added a Gaussian component to Model 2 and again fit the broadband spectrum. We found a $\chi^{2}$ (d.o.f.) of 3252(2794) and the $F$-test probability of chance 


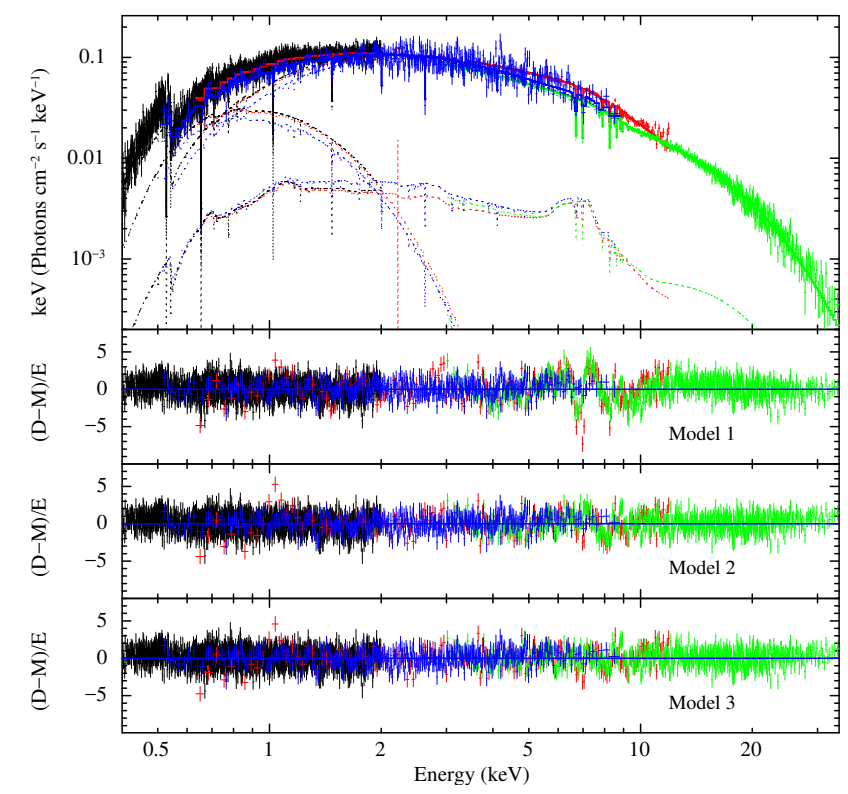

Fig. 3. High-flux spectrum and residuals corresponding to the models discussed in the text. The black, red, green, and blue data are associated with the RGS12, EPN, NuSTAR, and Swift/XRT spectra, respectively. From top to bottom: $E * f(E)$ is the unfolded spectrum associated with Model 3, and residuals in units of $\sigma$ ([data-model]/error) associated with Model 1, Model 2 and Model 3, respectively.

improvement was $2 \times 10^{-14}$. This shows that the addition of this component is statistically significant. The energy of the broad emission line is $6.60 \pm 0.10 \mathrm{keV}$, the width is $0.69 \pm 0.08 \mathrm{keV}$, and the normalization is $(1.1 \pm 0.1) \times 10^{-3}$ photons $\mathrm{cm}^{-2} \mathrm{~s}^{-1}$. These results are compatible within a $90 \%$ confidence level with those reported by Sidoli et al. (2001).

In the following we investigate a scenario in which the broad emission line is produced by reflection from the inner region of the accretion disk, where the relativistic effects smear the reflection component. We convolved the Comptonized component NTHCOMP with the reflection component RXFCONV (see Done \& Gierliński 2006; Kolehmainen et al. 2011, for details). We imposed in our model that the incident luminosity comes from the Comptonized component. The component RXFCONV has five parameters: the first is the redshift $z$, which we kept fixed to zero, the second is the iron abundance $\mathrm{Fe}_{\text {abundance, }}$, which we kept fixed to the solar abundance, the third is the cosine of the inclination angle $\theta$ of MXB 1659-298, which we assumed to be $72^{\circ}$ (Iaria et al. 2018), the fourth is the ionization parameter of the reflecting surface of the accretion disk $\log (\xi)$, which we left free to vary, and, finally, the fifth parameter is the relative reflection normalization of the component RXFCONV, measured in units of solid angle $\Omega / 2 \pi$ subtended by the reflector as seen from the corona ( $\mathrm{rel}_{\mathrm{refl}}$, hereafter).

However, the reflection region might be close to the NS, therefore we added the component RDBLUR to take into account the smearing associated with general and special relativistic effects. RDBLUR has four parameters: the inclination angle of the source, which we fixed at $72^{\circ}$ (see above), the inner and outer radius, $R_{\text {in }}$ and $R_{\text {out }}$, of the reflection region in units of gravitational radii $\left(G M / c^{2}\right.$, where $M$ is the mass of the compact object), and, finally, the power-law dependence of emissivity, Betor 10 .

In summary, Model 3 is composed of

Model $3=$ Edge $*$ TBabs $*($ partcov $*$ cabs $) *$ zxipcf $*($ diskbb + rdblur $* \operatorname{rfxconv} *$ nthComp $)$.
Initially, we left $R_{\text {in }}, R_{\text {out }}$, and Betor 10 free to vary. The value of $f_{\mathrm{IA}}$ shifts toward 1 , suggesting that the whole X-ray source is shielded by the ionized absorber. Hence we fixed the value of $f_{\mathrm{IA}}$ at 1 . Furthermore, because the value of $\chi^{2}$ is less sensitive to the changes of $R_{\text {out }}$, we fixed the value of $R_{\text {out }}$ at 2800 gravitational radii, which is the best-fit value of $R_{\text {out }}$.

A $\chi^{2}$ (d.o.f.) of 3228(2794) was obtained after the reflection component was added, whose statistical significance was confirmed by the $F$-test probability of chance improvement with respect to Model 2 equal to $7 \times 10^{-19}$. We also found that the self-consistent reflection model further improves the fit, resulting in a decrease of $\chi^{2}$ of 24 with respect to Model 2 plus broad line. We show the best-fit values in the sixth column of Table 1. The unfolded $E * f(E)$ spectrum and the corresponding residuals are shown in the top and bottom panels in Fig. 3, respectively.

We find that the equivalent hydrogen column density associated with the neutral interstellar matter is $(2.75 \pm 0.10) \times$ $10^{21} \mathrm{~cm}^{-2}$. The ionized absorbing matter has an equivalent hydrogen column density of $\left(6.0_{-1.4}^{+0.9}\right) \times 10^{23} \mathrm{~cm}^{-2}$, and its ionization parameter $\log (\xi)_{\text {IA }}$ was found equal to $4.37 \pm 0.04$ and $4.14 \pm 0.05$ for Obs. 2001 and Obs. 2016, respectively. The inner temperature of the multicolored disk emission is $0.30 \pm 0.02 \mathrm{keV}$ and the corresponding inner radius is $R_{\text {disk }} \sqrt{\cos \theta}=39 \pm 4 \mathrm{~km}$, assuming a distance of $10 \mathrm{kpc}$. The fit revealed that the powerlaw index and the electron and seed photon temperatures are all higher in Obs. 2016 than in Obs. 2001 (see Table 1).

The reflection component has a relative normalization of $0.22_{-0.05}^{+0.12}$, the inner radius of the reflecting region is $60_{-30}^{+60}$ gravitational radii, and the power-law dependence of emissivity is lower than -1.4 . Finally, the $0.4-35 \mathrm{keV}$ absorbed flux is $1.2 \times$ $10^{-9} \mathrm{erg} \mathrm{cm}^{-2} \mathrm{~s}^{-1}$, the extrapolated unabsorbed (excluding both the neutral and ionized absorber) flux in the $0.1-100 \mathrm{keV}$ energy range is $2.2 \times 10^{-9} \mathrm{erg} \mathrm{cm}^{-2} \mathrm{~s}^{-1}$, and the extrapolated unabsorbed flux in the $0.1-100 \mathrm{keV}$ energy range of the Comptonized component is $1.9 \times 10^{-9} \mathrm{erg} \mathrm{cm}^{-2} \mathrm{~s}^{-1}$. Assuming a distance of $10 \mathrm{kpc}$, the $0.1-100 \mathrm{keV}$ extrapolated luminosity of the Comptonized component is $2.2 \times 10^{37} \mathrm{erg} \mathrm{s}^{-1}$.

Model 3 assumes that the broadening of the emission line observed in the $\mathrm{Fe}-\mathrm{K}$ region of the spectrum is caused by both Compton scattering (included in the RFXCONV component) and relativistic smearing (included in the RDBLUR component). We checked whether the Compton scattering might by itself explain the width of the emission line by removing the component RDBLUR from Model 3. This seems not to be the case, as pointed out by the slightly higher $\chi^{2}$ (d.o.f.) of 3254(2796) with respect to the $\chi^{2}$ (d.o.f.) value of 3228(2794) found for Model 3 and the $F$-test probability of chance improvement of $1.4 \times 10^{-5}$, corresponding to a statistical improvement larger than $4 \sigma$.

The RGS12 and EPN spectrum showed clear signatures of absorption lines at $0.65 \mathrm{keV}, 1 \mathrm{keV}$, and in the $\mathrm{Fe}-\mathrm{K}$ region. We show in Fig. 4 the RGS12 spectrum in the $0.5-1.1 \mathrm{keV}$ energy range and the corresponding residuals obtained by fitting the data with a power-law component with a photon index of $1.25 \pm 0.04$, absorbed by neutral interstellar matter with an equivalent hydrogen column density of $(0.302 \pm$ $0.008) \times 10^{22} \mathrm{~cm}^{-2}$. We considered the RGS12 energy range between 0.62 and $0.70 \mathrm{keV}$ and fit the continuum emission by adopting a power-law component. Then we added a Gaussian line with negative normalization to fit the absorption line at $0.65 \mathrm{keV}$, and we fixed the value of the power-law index to its best-fit value to estimate the uncertainties for the absorption line parameters. The best-fit parameters of the Gaussian absorption lines are shown in Table 2. Using the RGS12 energy range 


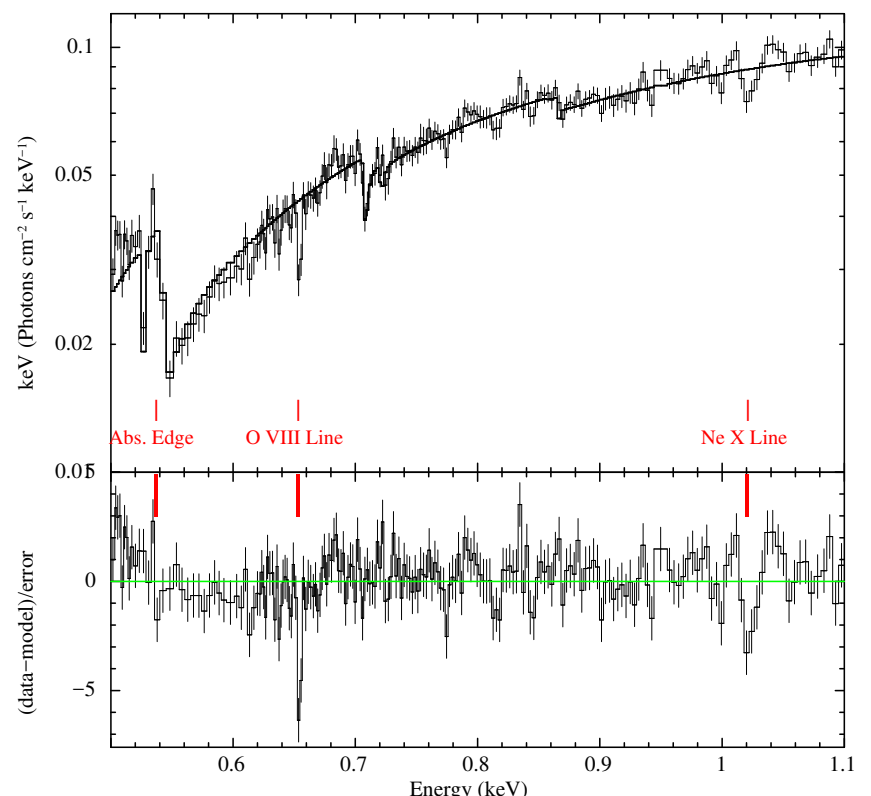

Fig. 4. High-flux RGS12 spectrum and residuals corresponding to the models composed of a power-law component absorbed by neutral interstellar matter. We marked the energies at $0.54,0.64$, and $1.022 \mathrm{keV}$ that correspond to the absorption edge associated with neutral oxygen and to the absorption lines associated with $\mathrm{O}$ VIII and $\mathrm{Ne} \mathrm{X}$, respectively.

between 0.98 and $1.04 \mathrm{keV}$ and adopting the same approach described above, we found the best-fit parameters of the Gaussian absorption line associated with the $\mathrm{Ne} \mathrm{X} \mathrm{Ly}_{\alpha}$ transition. We selected the EPN energy range between 6.4 and $7.5 \mathrm{keV}$ and found the best-fit parameters of the Gaussian absorption lines to be associated with the resonant transition of Fe XXV line and to the Fe XXVI Ly $\mathrm{L}_{\alpha}$ line. Finally, selecting the 7.4-8.5 keV energy range, we estimated the best-fit values of the Gaussian absorption lines associated with the $\mathrm{Ly}_{\beta}$ transitions of the Fe XXV and Fe XXVI lines. All these results are shown in Table 2.

\subsection{Low-flux spectrum (the hard state)}

We used the same Model 1 as we adopted for the high-flux spectrum, but the DISKBB component is not required here. To fit the low-flux spectrum, we also added a Gaussian emission line at $2.22 \mathrm{keV}$ to fit the residuals of the EPN spectrum between 1.8 and $2.4 \mathrm{keV}$. The first model in this case is

Model $1=$ TBabs $*$ nthComp.

We obtained a $\chi^{2}$ (d.o.f.) value of $2967(2278)$. We show the bestfit parameters of Model 1 in the third column of Table 3, and the residuals are shown in the second panel from the top in Fig. 5. The residuals show absorption features between 6 and $9 \mathrm{keV}$. For this reason we added the ZXIPCF component. The new model is

Model $2=$ TBabs $*($ partcov $*$ cabs $)$

$$
* \text { zxipcf } * \text { nthComp. }
$$

We left the ionization parameter associated with the XMMNewton and NUSTAR spectrum free to vary independently. Fitting the spectra with Model 2, we obtained a $\chi^{2}$ (d.o.f.) value of 2556(2274). The addition of the ZXIPCF component is statistically significant (the $F$-test probability of chance improvement is $4 \times 10^{-72}$ ). We show the best-fit parameters in the fourth column of Table 3 . However, we observe that large residuals are still present above $10 \mathrm{keV}$ in the NUSTAR spectrum (see the third panel from the top of Fig. 5). These might be associated with a Compton hump caused by reflection from the accretion disk. Similarly to the case of the high-flux spectrum, we added a reflection component smeared by relativistic effects. The adopted model was

$$
\begin{aligned}
\text { Model } 3= & \text { TBabs } *(\text { partcov } * \text { cabs }) \\
& * \text { zxipcf } * \text { rdblur } * \operatorname{rfxconv} * \text { nthComp. }
\end{aligned}
$$

The value of $\chi^{2}$ is less sensitive to change in $R_{\text {out }}$, hence we fixed the value of $R_{\text {out }}$ to the best-fit value of 290 gravitational radii. We obtained a $\chi^{2}$ (d.o.f.) value of 2359(2270). The addition of the reflection component is statistically significant, with an obtained $F$-test probability of chance improvement of $3 \times 10^{-38}$. We show the best-fit values in the fifth column of Table 3 , and the unfolded $E * f(E)$ spectrum with the model components and the corresponding residuals are shown in the top and bottom panels in Fig. 5, respectively.

We refit the spectrum by fixing the value of $R_{\text {out }}$ to the value obtained for the high-flux spectrum (2800 gravitational radii); the best-fit values of the other parameters did not change. However, the $\chi^{2}$ value is higher and $\Delta \chi^{2}$ is 4 , therefore we discuss our results with $R_{\text {out }}$ fixed at 290 gravitational radii.

We obtained that the equivalent hydrogen column associated with the neutral interstellar matter is $N_{\mathrm{H}_{\text {ISM }}}=\left(3.29_{-0.08}^{+0.04}\right) \times$ $10^{21} \mathrm{~cm}^{-2}$. The ionized absorbing matter around the system has an equivalent hydrogen column of $N_{\mathrm{H}}=(1.41 \pm 0.12) \times 10^{23} \mathrm{~cm}^{-2}$ and it covers $(25 \pm 2) \%$ of the emitting source. The value of $\log (\xi)_{\text {IA }}$ is $1.98 \pm 0.05$ and $3.1 \pm 0.3$ for the XMM-Newton and NuSTAR observation, respectively. The Comptonized component is hard, with an electron temperature $k T_{\mathrm{e}}$ higher than $130 \mathrm{keV}$ and a seed-photon temperature $k T_{\text {bb }}$ lower than $0.06 \mathrm{keV}$; the photon index is $\Gamma=1.989 \pm 0.015$.

The best-fit values for $\log (\xi)$ and $\operatorname{rel}_{\text {refl }}\left(1.99_{-0.10}^{+0.05}\right.$ and $0.48 \pm$ 0.06 , respectively) suggest that the reflecting region probably lies close the NS and that the Comptonized corona may have a slab geometry. The power-law dependency of emissivity is Betor $10=-3.0 \pm 0.3$, and the inner radius of the reflecting region is smaller than seven gravitational radii.

Finally, the $0.45-55 \mathrm{keV}$ absorbed flux is $2 \times 10^{-10} \mathrm{erg} \mathrm{cm}^{-2} \mathrm{~s}^{-1}$ and the extrapolated unabsorbed (excluding both the neutral and ionized absorber) flux in the $0.1-100 \mathrm{keV}$ energy range is $4.4 \times 10^{-10} \mathrm{erg} \mathrm{cm}^{-2} \mathrm{~s}^{-1}$. The extrapolated unabsorbed flux in the $0.1-100 \mathrm{keV}$ energy range of the Comptonized component is $4 \times 10^{-10} \mathrm{erg} \mathrm{cm}^{-2} \mathrm{~s}^{-1}$ and the corresponding luminosity is $4 \times$ $10^{36} \mathrm{erg} \mathrm{s}^{-1}$ for a distance to the source of $10 \mathrm{kpc}$.

\section{Discussion}

We have analyzed the high-flux and low-flux spectra of MXB 1659-298 collected during the 1999 and 2015 outbursts. We find that the high-flux spectrum shows a soft Comptonized component with a value of the electron temperature lower than $4 \mathrm{keV}$. Furthermore, a multicolor disk blackbody component is present at low energies with an inner temperature of $0.30 \mathrm{keV}$ and an inner radius of the disk of $R_{\mathrm{in}} \sqrt{\cos \theta}=39 \pm 4 \mathrm{~km}$ (assuming a distance to the source of $10 \mathrm{kpc}$ ). The low-flux spectrum has a hard Comptonized component with a value of the electron temperature higher than $130 \mathrm{keV}$, while the addition of a multicolor disk component is not statistically significant.

According to the fits, in both observations the source is (at least partially) covered by ionized absorbing matter. In particular, the covering by this absorbing material is complete in 
Table 2. Best-fit parameters of the absorption lines in the high-flux spectrum.

\begin{tabular}{lcccccc}
\hline \hline \multirow{2}{*}{$\begin{array}{l}\text { Ion and } \\
\text { transition }\end{array}$} & $\begin{array}{c}\text { Measured } \\
\text { energy }(\mathrm{keV})\end{array}$ & $\begin{array}{c}\text { Theoretical } \\
\text { energy }(\mathrm{keV})\end{array}$ & $\begin{array}{c}\mathrm{EW} \\
(\mathrm{eV})\end{array}$ & $\begin{array}{c}\text { Intensity } \\
\left(10^{-4} \mathrm{ph} \mathrm{cm}^{-2} \mathrm{~s}^{-1}\right)\end{array}$ & \begin{tabular}{c} 
Line width \\
\cline { 5 - 7 }$(\mathrm{eV})$
\end{tabular} & $\left(\mathrm{km} \mathrm{s}^{-1}\right)$ \\
\hline O VIII 1s-2p & $0.6540 \pm 0.0003$ & 0.6536 & $-1.8_{-0.4}^{+0.3}$ & $-1.2 \pm 0.2$ & $<1.2$ & $<550$ \\
Ne X 1s-2p & $1.0216_{-0.0011}^{+0.0019}$ & 1.0218 & $-3.6 \pm 1.0$ & $-3.4_{-0.5}^{+0.7}$ & $2.8_{-0.8}^{+1.3}$ & $820_{-230}^{+380}$ \\
Fe XXV 1s ${ }^{2}-1 \mathrm{~s} 2 \mathrm{p}$ & $6.705_{-0.0013}^{+0.0160}$ & 6.700 & $-34_{-4}^{+6}$ & $-2.5 \pm 0.3$ & $<42$ & $<1900$ \\
Fe XXVI 1s-2p & $6.992_{-0.004}^{+0.015}$ & 6.966 & $-46_{-3}^{+5}$ & $-3.1 \pm 0.3$ & $<31$ & $<1300$ \\
Fe XXV 1s ${ }^{2}-1 \mathrm{~s} 3 \mathrm{p}$ & $7.91_{-0.04}^{+0.06}$ & 7.88 & $-33 \pm 11$ & $-1.6_{-0.7}^{+0.5}$ & $<190$ & $<7200$ \\
Fe XXVI 1s-3p & $8.26 \pm 0.03$ & 8.25 & $-45 \pm 10$ & $-1.9 \pm 0.5$ & $<130$ & $<4700$ \\
\hline
\end{tabular}

Notes. Uncertainties are given at $68 \%$ c.l., upper limits at $90 \%$ c.l. The best-fit values of the energies are compatible with the rest-frame values at $90 \%$ c.l.

Table 3. Best-fit parameters of the low-flux spectrum.

\begin{tabular}{|c|c|c|c|c|c|c|c|}
\hline \multirow[t]{2}{*}{ Model } & \multirow[t]{2}{*}{ Component } & \multicolumn{2}{|c|}{ Model 1} & \multicolumn{2}{|c|}{ Model 2} & \multicolumn{2}{|c|}{ Model 3} \\
\hline & & XMM-Newton & NUSTAR & XMM-Newton & NuSTAR & XMM-Newton & NUSTAR \\
\hline \multirow[t]{3}{*}{ ZXIPCF } & $N_{\mathrm{H}}\left(10^{22}\right.$ atoms $\left.\mathrm{cm}^{-2}\right)$ & \multicolumn{2}{|l|}{-} & \multicolumn{2}{|c|}{$13 \pm 2$} & \multicolumn{2}{|c|}{$14.1 \pm 1.2$} \\
\hline & $\log (\xi)_{\mathrm{IA}}$ & \multirow{2}{*}{\multicolumn{2}{|c|}{$\begin{array}{l}- \\
-\end{array}$}} & $1.09_{-028}^{+0.12}$ & \multirow{2}{*}{$2.9 \pm 0.2$} & \multicolumn{2}{|c|}{$1.98 \pm 0.05 \quad 3.1 \pm 0.3$} \\
\hline & $f_{\mathrm{IA}}$ & & & \multirow{2}{*}{\multicolumn{2}{|c|}{$\begin{array}{l}0.21 \pm 0.02 \\
0.237^{+0.007}\end{array}$}} & \multicolumn{2}{|c|}{$0.25 \pm 0.02$} \\
\hline TBABS & $N_{\mathrm{H}_{\text {ISM }}}\left(10^{22}\right.$ atoms $\left.\mathrm{cm}^{-2}\right)$ & \multicolumn{2}{|c|}{$0.231 \pm 0.005$} & & & \multicolumn{2}{|c|}{$0.329_{-0.008}^{+0.004}$} \\
\hline PARTCOV & $f$ & \multicolumn{2}{|c|}{-} & \multicolumn{2}{|c|}{$0.21 \pm 0.02$} & \multicolumn{2}{|c|}{$0.25 \pm 0.02$} \\
\hline CABS & $N_{\mathrm{H}}\left(10^{22}\right.$ atoms $\left.\mathrm{cm}^{-2}\right)$ & \multicolumn{2}{|l|}{-} & \multicolumn{2}{|c|}{$13 \pm 2$} & \multicolumn{2}{|c|}{$14.1 \pm 1.2$} \\
\hline RDBLUR & Betor10 & \multicolumn{2}{|l|}{-} & \multicolumn{2}{|c|}{-} & \multicolumn{2}{|c|}{$-3.0 \pm 0.3$} \\
\hline & $R_{\text {in }}\left(G M / c^{2}\right)$ & \multicolumn{2}{|l|}{-} & \multicolumn{2}{|l|}{-} & \multicolumn{2}{|c|}{$<7$} \\
\hline & $R_{\text {out }}\left(G M / c^{2}\right)$ & \multicolumn{2}{|l|}{-} & \multicolumn{2}{|l|}{-} & \multicolumn{2}{|c|}{290 (frozen) } \\
\hline & $\theta(\operatorname{deg})$ & \multicolumn{2}{|l|}{-} & \multicolumn{2}{|l|}{-} & 72 (fro & \\
\hline RFXCONV & $\mathrm{rel}_{\text {refl }}$ & \multicolumn{2}{|l|}{-} & \multicolumn{2}{|l|}{-} & $0.48 \pm$ & 06 \\
\hline & $\mathrm{Fe}_{\text {abund }}$ & - & & - & & 1 (froz & \\
\hline & $\cos \theta$ & - & & - & & $0.309(\mathrm{fr}$ & zen) \\
\hline & $\log (\xi)$ & - & & - & & $1.99_{-c}^{+c}$ & \\
\hline NTHCOMP & $\Gamma$ & $1.842 \pm 0$ & 004 & $1.911 \pm($ & 011 & $1.989 \pm$ & 015 \\
\hline & $\mathrm{kT}_{\mathrm{e}}(\mathrm{keV})$ & $37_{-11}^{+3}$ & & $>180$ & & $>13$ & \\
\hline & $\mathrm{kT}_{\mathrm{bb}}(\mathrm{keV})$ & $0.113 \pm 0$ & 007 & $0.120 \pm$ & 009 & $<0.0$ & \\
\hline & norm & $0.0242 \pm 0$ & 0003 & $0.0318 \pm$ & 0006 & $0.0343 \pm$ & 0011 \\
\hline & $\chi^{2} /$ d.o.f. & $2967 / 2$ & & $2556 / 2$ & & $2359 / 2$ & \\
\hline
\end{tabular}

the soft state, where the equivalent hydrogen column density is found equal to $\left(60_{-14}^{+9}\right) \times 10^{22} \mathrm{~cm}^{-2}$, and only partial in the hard state, with a covering fraction of $25 \%$. The ionization parameter $\log (\xi)_{\mathrm{IA}}$ of the absorbing material is higher than 4 in the highflux state and lower than 3.1 in the low-flux state.

The spectra both show a reflection component from the accretion disk. We find that when we take the relativistic smearing into account by adding the RDBLUR component to the reflection component, we improve the fit with a statistical significance higher than $4 \sigma$. This suggests that the broad width of the emission line observed in the $\mathrm{Fe}-\mathrm{K}$ region cannot be explained invoking only the Compton broadening, but it is necessary to also take the relativistic smearing into account.

The reflecting skin above the disk has an ionization parameter $\log (\xi)$ of $2.80_{-0.10}^{+0.20}$ and $1.99_{-0.10}^{+0.05}$ in the soft and hard state, respectively. The reflecting region is between $39_{-15}^{+35}$ and 2800 gravitational radii in the high state, and it is closer to the NS surface in the low state, for which its boundaries are smaller than 7 and 290 gravitational radii, respectively. A similar behavior of the reflection region was observed by Mazzola et al. (2019) in soft and high state of the NS-LMXB 4U 1702-429 and by Di Salvo et al. (2015) in 4U 1705-44 (see also Egron et al. 2013).

Assuming an NS mass of $1.54 M_{\odot}$ (see Özel \& Freire 2016), the reflecting region in the high state is between $140_{-70}^{+140} \mathrm{~km}$ and $6.4 \times 10^{3} \mathrm{~km}$. The inner radius of the accretion disk is $R_{\text {disk }}=70 \pm 7 \mathrm{~km}$, under the assumption that the inclination angle of MXB 1659-298 is $72^{\circ}$. However, because the inferred luminosity (see Sect. 3.1) is $10 \%$ of the Eddington luminosity, the effective inner radius of the accretion disk $r_{\text {disk }}$ is given by the relation $r_{\text {disk }}=s^{2} R_{\text {disk }}$ where $s=1.7$ (see Shimura \& Takahara 1995). We obtain $r_{\text {disk }}=200 \pm 20 \mathrm{~km}$, which is compatible to the boundaries of the reflecting region estimated above.

We estimated the optical depth of the Comptonized cloud for the high and soft state using the relation between the powerlaw photon index $\Gamma$ and the electron temperature $k T_{\mathrm{e}}$ obtained by Zdziarski et al. (1996). As expected, we find that the Comptonizing cloud is optically thick in the soft state ( $\tau$ is $15.7 \pm 0.5$ and $8.0 \pm 0.2$ for the spectra taken in 2001 and 2016, respectively), and it is optically thin in the hard state $(\tau \lesssim 0.7)$.

We determined the average electron number density $n_{\mathrm{e}}$ of the Comptonized cloud using the relation $\tau=\sigma_{\mathrm{T}} n_{\mathrm{e}} d$, where $\sigma_{\mathrm{T}}$ 


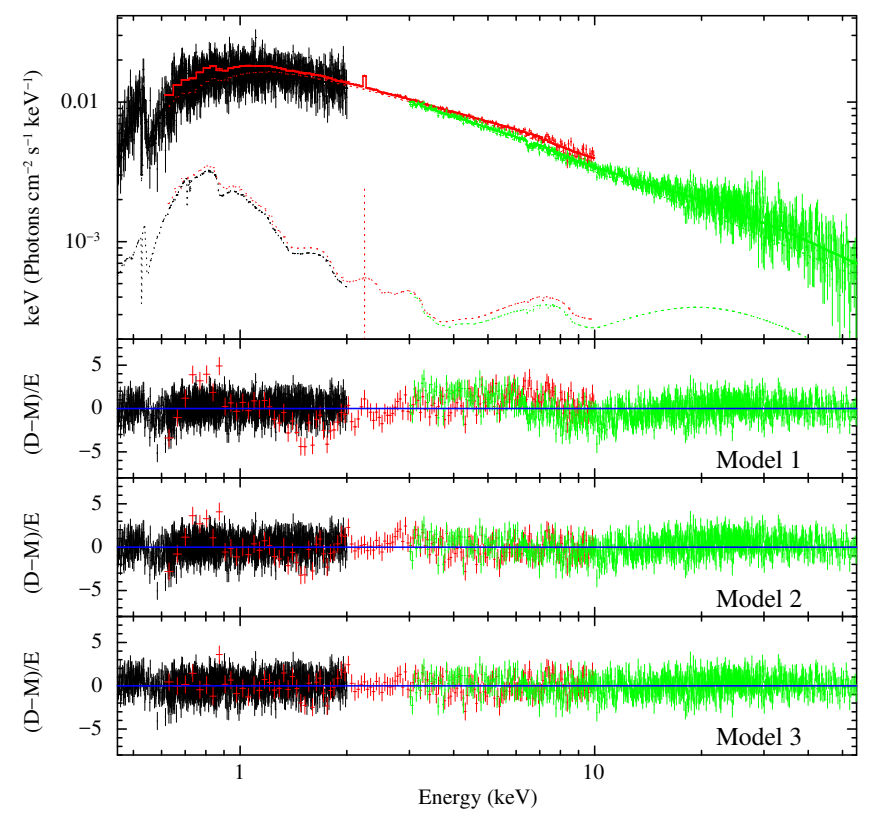

Fig. 5. Low-flux spectrum and residuals corresponding to the models discussed in the text. The colors are defined as in Fig. 3.

is the Thomson cross-section and $\mathrm{d}$ is the geometrical dimension of the cloud. In the case of the soft state we assumed that $\mathrm{d}$ is equal to $r_{\text {disk }}$, which is plausible because the disk emission from the innermost region could be occulted by the optically thick Comptonized cloud surrounding the compact object. We found $n_{\mathrm{e}}=(9 \pm 2) \times 10^{17} \mathrm{~cm}^{-3}$ and $n_{\mathrm{e}}=(5 \pm 1) \times 10^{17} \mathrm{~cm}^{-3}$ for the soft state during the observation taken in 2001 and 2016, respectively. An upper limit on the average electron number density $n_{\mathrm{e}}$ of the Comptonized cloud in the hard state can be inferred assuming that the size of the Comptonized cloud has to be smaller than the outer radius of the reflecting skin $(290$ gravitational radii, corresponding to $670 \mathrm{~km}$ for an NS mass of $1.54 M_{\odot}$ ), assuming that the Comptonized cloud does not fully cover the reflecting region. Knowing that the optical depth associated with the Comptonized cloud is lower than 0.7, we obtain $n_{\mathrm{e}}<2 \times 10^{16} \mathrm{~cm}^{-3}$ at a distance of $670 \mathrm{~km}$ from the NS.

On the other hand, the electron number density $n_{\mathrm{e}}$ associated with the reflecting skin can be inferred taking into account that $\log (\xi) \simeq 2.8$, because $\xi=L_{\mathrm{X}} /\left(n_{\mathrm{e}} r^{2}\right)$, where $L_{\mathrm{X}}$ is the incident luminosity and $r$ is the distance between the X-ray source and the reflecting region. The incident luminosity $L_{\mathrm{X}}$ associated with the Comptonized component is $2.4 \times 10^{37} \mathrm{erg} \mathrm{s}^{-1}$ in the high state, and $r$ is roughly between $140_{-70}^{+140} \mathrm{~km}$ and $6.4 \times 10^{3} \mathrm{~km}$. By substituting these values, we infer that the electron number density of the reflecting skin is between $\sim 2 \times 10^{20}$ and $\sim 9 \times 10^{16} \mathrm{~cm}^{-3}$ going from $R_{\text {in }}$ to $R_{\text {out }}$; at the distance of $r_{\text {disk }}$, we find that $n_{\mathrm{e}} \simeq 1.0 \times 10^{20} \mathrm{~cm}^{-3}$. The electron number density $n_{\mathrm{e}}$ associated with the reflecting skin in the low state is between $\sim 4 \times 10^{22}$ and $\sim 1 \times 10^{19} \mathrm{~cm}^{-3}$ going from the NS surface to $R_{\text {out }}=670 \mathrm{~km}$.

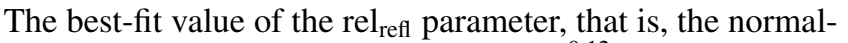
ization of the RXFCONV component, is $0.22_{-0.05}^{+0.12}$ and $0.48 \pm 0.06$ for the high-flux spectrum (optically thick corona) and lowflux spectrum (optically thin corona), respectively. These values describe a scenario well in which a spherical corona is present in the inner part of the accretion disk (see Fig. 5 in Dove et al. 1997). The reflecting radius is a factor 1.2 larger than the coronal radius in the high-flux state, and the two radii are compatible with each other in the low-flux state.
We detect an ionized absorber in both the high and low state. Assuming roughly that $n_{\mathrm{e}}=n_{\mathrm{H}}$, we expect that the absorbing ionized cloud is optically thin. Solving $\tau=\sigma_{\mathrm{T}} n_{\mathrm{e}} d=\sigma_{\mathrm{T}} n_{\mathrm{H}} d=$ $\sigma_{\mathrm{T}} N_{\mathrm{H}}$, we obtained $\tau \simeq 0.4$ and $\tau \simeq 0.09$ for the soft and hard state, respectively. To describe the ionized absorber, we adopted the model ZXIPCF and fit the data assuming a unique value of the ionization parameter, unlike Sidoli et al. (2001), who suggested that the ionized matter around MXB 1659-298 should have a gradient of ionization to explain the simultaneous presence of absorption lines associated with heavy ions such as Fe XXV and Fe XXVI and light ions such as O VIII and NeX. However, we observe that the model fits all the absorption lines well that are detected in the spectrum, suggesting that an absorber with a unique ionization parameter cannot be excluded. In this scenario we estimate the location of the ionized absorbing matter and its thickness.

In the soft state we observe that the equivalent hydrogen column density associated with the ionized absorbing matter is $\left(6.0_{-0.8}^{+0.4}\right) \times 10^{23} \mathrm{~cm}^{-2}$ (uncertainties at $68 \%$ c.l.). Using the cosmic abundance for oxygen, neon, and iron reported by Wilms et al. (2000) (i.e., $4.9 \times 10^{-4}, 8.7 \times 10^{-5}$ and $2.7 \times 10^{-5}$, respectively), we evaluate the equivalent column density for the three elements $N_{\mathrm{O}}=\left(2.8_{-0.6}^{+0.3}\right) \times 10^{20} \mathrm{~cm}^{-2}, N_{\mathrm{Ne}}=\left(5.0_{-1.1}^{+0.5}\right) \times 10^{19} \mathrm{~cm}^{-2}$, and $N_{\mathrm{Fe}}=\left(1.5_{-0.4}^{+0.2}\right) \times 10^{19} \mathrm{~cm}^{-2}$. Assuming that the ionized matter is illuminated by a power law with spectral index $\Gamma=2$, we expect for an ionization parameter $\log _{\xi}=4.36$ that the abundance of FeXXV and FeXXVI ions with respect to the neutral iron is $f_{\mathrm{Fexxv}} \simeq 0.05$ and $f_{\mathrm{FexxvI}} \simeq 0.34$ (see Kallman \& Bautista 2001). Using these abundances, we find that the equivalent column densities associated with Fe XXV and Fe XXVI are $N_{\mathrm{Fexxv}}=$ $\left(7.5_{-2.0}^{+1.0}\right) \times 10^{17} \mathrm{~cm}^{-2}$ and $N_{\mathrm{FexxvI}}=\left(5.1_{-1.4}^{+0.7}\right) \times 10^{18} \mathrm{~cm}^{-2}$, respectively.

We obtain a relation between the observed equivalent widths of the absorption lines and the kinetic temperature of the plasma using Eqs. (1)-(9) shown by Kotani et al. (2000) to estimate the curves of growth of the observed ions. For this aim, we adopt the Einstein coefficients and the values of the oscillator strengths reported by Verner et al. (1996b). We find that the kinetic temperatures associated with the Fe XXVI and Fe XXV ions are $k T=$ $100_{-30}^{+45} \mathrm{keV}$ and $k T=130_{-70}^{+470} \mathrm{keV}$, respectively. These temperatures are compatible with each other, therefore it is possible to assume that the Fe XXVI and Fe XXV ions are present in the same region of the ionized absorber and the kinetic temperature associated with the highly ionized iron is $k T_{\text {kin }}=100_{-30}^{+45} \mathrm{keV}$.

When we assume that the ionized matter is illuminated by a radiation described as a power law with spectral index $\Gamma=2$, the thermal temperature of the photoionized matter is $k T_{\text {th }}=$ $7.1_{-0.1}^{+0.2} \mathrm{keV}$ for an ionization parameter of $\log (\xi)_{\mathrm{IA}}=4.37 \pm 0.04$ (Kallman et al. 2004). The relation between the thermal and kinetic temperature is given by Eq. (1) of Yamaoka et al. (2001):

$k T_{\text {th }}+\frac{1}{3} m_{\mathrm{Fe}} v_{\text {bulk }}^{2}=k T_{\text {kin }}$,

where $m_{\mathrm{Fe}}$ is the mass of an iron atom and $v_{\text {bulk }}$ denotes the velocity of bulk motion. Yamaoka et al. (2001) proposed two different scenarios for large bulk motions corresponding to the $k T_{\text {kin }}$ range of $50-500 \mathrm{keV}$. The first possibility consists of randomly oriented turbulent motions in the plasma, and the second attributes the velocity dispersion to the radial velocity gradient. In the second case the ionized absorber may undergo an outflow, such as a radiation-driven wind. However, we exclude the second scenario because the observed absorption lines have energies that are compatible with the rest frame value. Furthermore, 
Díaz Trigo \& Boirin (2016) suggested that MXB 1659-298 could have a mild thermal wind, even if only static atmospheres have been reported so far.

Using the values of $k T_{\text {kin }}=100_{-30}^{+45} \mathrm{keV}$ and $k T_{\text {th }}=7.1_{-0.1}^{+0.2} \mathrm{keV}$, we obtain that the turbulent velocity is $v_{\text {bulk }}=690_{-110}^{+170} \mathrm{~km} \mathrm{~s}^{-1}$. This value is not compatible with the value of $200 \mathrm{~km} \mathrm{~s}^{-1}$ adopted using the model ZXIPCF, and it can explain why the widths of the absorption lines in the $\mathrm{Fe}-\mathrm{K}$ region are not well fit.

A turbulent velocity (associated with the ionized iron) higher than $500 \mathrm{~km} \mathrm{~s}^{-1}$ was also observed in the eclipsing NS binary system AX J1745.6-2901 (Ponti et al. 2015). Using the values shown in Table 2, we estimate the flux ratio $R$ between the $\mathrm{K} \alpha$ and $\mathrm{K} \beta$ transitions for the Fe XXV and Fe XXVI absorption lines and find $R=1.6_{-0.6}^{+0.8}$ and $R=1.6 \pm 0.6$, respectively. Figure 4 of Risaliti et al. (2005) shows that the obtained values of $R$ give a lower limit on the turbulent velocity of $100 \mathrm{~km} \mathrm{~s}^{-1}$, which is compatible with a turbulent velocity between 500 and $1000 \mathrm{~km} \mathrm{~s}^{-1}$ for an equivalent column density of neutral hydrogen of $\left(6.0_{-1.4}^{+0.9}\right) \times 10^{23} \mathrm{~cm}^{-2}$.

Assuming that the plasma is in hydrodynamical equilibrium in the vertical direction to the disk plane, the distance $r_{\mathrm{Fe}}$ from the central source of the absorbing plasma containing highly ionized iron can be estimated from its thermal temperature using the following expression:

$\left(h / r_{\mathrm{Fe}}\right)^{2} \times\left(G M \mu / r_{\mathrm{Fe}}\right)=k T_{\mathrm{th}}$,

(see Sidoli et al. 2001, and references therein), where $M$ is the NS mass, $\mu \simeq\left(0.61 m_{\mathrm{H}}\right)$ is the mean atomic mass of the matter assuming that it is fully ionized, $m_{\mathrm{H}}$ is the mass of an hydrogen atom, $G$ is the gravitational constant, and $h$ is the scale height of the absorbing plasma. Assuming that $h / r_{\mathrm{Fe}}=\tan \theta=\tan (\pi / 2-i)$, where $i$ is the inclination angle of source, adopting an inclination angle of $72^{\circ} \pm 3^{\circ}$ and an NS mass of $1.54 \pm 0.22 M_{\odot}$, we obtain $r_{\mathrm{Fe}}=(1.9 \pm 0.7) \times 10^{9} \mathrm{~cm}$.

The electron density $n_{\mathrm{e}}$ in which the iron lines originate can be estimated using the expression $n_{\mathrm{e}}=L_{\mathrm{X}} /\left(\xi r_{\mathrm{Fe}}^{2}\right)$, where $L_{\mathrm{X}}$ is the luminosity of the source and $\xi$ is the ionization parameter obtained from the fit. We find that $n_{\mathrm{e}}=(3.3 \pm 1.3) \times 10^{14} \mathrm{~cm}^{-3}$, assuming an uncertainty of $10 \%$ associated with the luminosity $L_{\mathrm{X}}$. We estimated the thickness $\Delta r$ of the absorbing plasma using the expression $N_{\mathrm{H}}=n_{\mathrm{e}} \Delta r$, under the assumption that $n_{\mathrm{e}} \simeq n_{\mathrm{H}}$ and adopting the value of $N_{\mathrm{H}}$ obtained from the best fit (see Table 1). In this way, we obtained $\Delta r=\left(1.7_{-0.8}^{+0.7}\right) \times 10^{9} \mathrm{~cm}$.

Sidoli et al. (2001) suggested that the O VIII and Ne IX absorption lines are produced at larger radii than the iron ionized absorption lines by invoking a gradient of the ionization parameter. The obtained velocities, associated with the broadening of the lines shown in the seventh column of Table 2, are due to both the thermal broadening and the turbulent velocity. When the line-of-sight turbulent velocity distribution is described as Gaussian, the combined velocity $v_{\mathrm{D}}$ is defined as

$v_{\mathrm{D}}^{2}=v_{\mathrm{th}}^{2}+v_{\mathrm{bulk}}^{2}$,

where $v_{\text {th }}$ and $v_{\text {bulk }}$ are the thermal and turbulent velocities, respectively. The thermal velocities is expressed as

$v_{\mathrm{th}}^{2}=2 \frac{k T_{\mathrm{th}_{\mathrm{I}}}}{m_{\mathrm{I}}}$,

where $k T_{\mathrm{th}_{\mathrm{I}}}$ is the thermal energy of the absorber and $m_{\mathrm{I}}$ the mass of the atom that we considered. Using the estimated values associated to the ionized iron and assuming that the absorber is in hydrodynamical equilibrium along the vertical direction, we can write

$\frac{r_{\mathrm{I}}}{r_{\mathrm{Fe}}}=\frac{k T_{\mathrm{th}_{\mathrm{Fe}}}}{k T_{\mathrm{th}_{\mathrm{I}}}}$,

where $r_{\mathrm{Fe}}$ and $r_{\mathrm{I}}$ are the distances from the NS where the corresponding lines form. Coronal models tend to have turbulent velocities that are locally proportional to the virial or rotational velocity (Woods et al. 1996; Iaria et al. 2007), then we can write

$\frac{r_{\mathrm{I}}}{r_{\mathrm{Fe}}}=\left(\frac{v_{\text {bulk }_{\mathrm{Fe}}}}{v_{\text {bulk }_{\mathrm{I}}}}\right)^{2}$,

where $v_{\text {bulk }}$ and $v_{\text {bulk }}$ are the turbulent velocities of the absorber where the ionized iron lines and the light ion lines form, respectively. Combining Eqs. (3)-(6), we obtain a relation between the thermal energy of the absorber and the observed velocity:

$k T_{\mathrm{th}_{\mathrm{I}}}=\frac{s}{1+s\left(v_{\mathrm{bulk}_{\mathrm{Fe}}}^{2} / k T_{\mathrm{th}_{\mathrm{Fe}}}\right)} v_{\mathrm{D}}^{2} \mathrm{keV}$,

where $s=511 / 2\left(m_{\mathrm{I}} / m_{\mathrm{e}}\right) 1 / c^{2}$ with $m_{\mathrm{e}}$ the electron mass and $c$ the speed of light.

Using the best values of the thermal energy and turbulent velocity associated with the ionized iron (i.e., $k T_{\text {th }_{\mathrm{Fe}}} \simeq 7.1 \mathrm{keV}$ and $v_{\text {bulk }} \simeq 690 \mathrm{~km} \mathrm{~s}^{-1}$, respectively), we find the thermal energy as a function of the velocity $v_{\mathrm{D}}$ for the $\mathrm{Ne} \mathrm{X}$ absorption line that is shown in Fig. 6. We expect that if the $\mathrm{Ne} \mathrm{X}$ absorption line is produced in an outer region with respect to the region where the ionized iron lines are formed, then the thermal energy of the absorber should be lower than $7.1 \mathrm{keV}$. We find that the $\mathrm{NeX}$ absorption line could be produced in an absorber with a thermal temperature between 4.5 and $7.1 \mathrm{keV}$, corresponding to a value of $v_{\mathrm{D}}$ of 590 and $738 \mathrm{~km} \mathrm{~s}^{-1}$, respectively. Using the equations reported above, we find that the $\mathrm{Ne} \mathrm{X}$ absorption line forms between $r=(1.9 \pm 0.7) \times 10^{9} \mathrm{~cm}$ and $r=(3.0 \pm 1.1) \times 10^{9} \mathrm{~cm}$, respectively, and the kinetic energy is between 25.6 and $40 \mathrm{keV}$. Furthermore, the spanning range of distances $r$ is compatible with the thickness of the absorber $\Delta r=\left(1.7_{-0.8}^{+0.7}\right) \times 10^{9} \mathrm{~cm}$ estimated for the ionized iron lines, suggesting that the ionized iron lines and the $\mathrm{Ne} \mathrm{X}$ absorption line could be produced in the same region of the absorber.

Using Eqs. (1)-(9) of Kotani et al. (2000), we estimate that the fraction $f$ of $\mathrm{NeX}$ ions compared with the whole population of $\mathrm{Ne}$ ions from the information that the equivalent column density of neutral neon atoms is $N_{\mathrm{Ne}}=\left(5.0_{-1.1}^{+0.5}\right) \times 10^{19} \mathrm{~cm}^{-2}$ and that the measured equivalent width of the $\mathrm{Ne} X$ absorption line is $-3.6 \pm 1.0 \mathrm{eV}$. We obtain that $f=\left(3_{-2}^{+4}\right) \times 10^{-3}$ and $f=\left(2.5_{-1.0}^{+2.5}\right) \times 10^{-3}$ for a thermal energy between 4.5 and $7.1 \mathrm{keV}$, respectively, corresponding to an equivalent column density associated with $\mathrm{Ne} X$ ions of $1.5 \times 10^{17} \mathrm{~cm}^{-2}$.

Regarding the O VIII absorption line, we show the thermal energy as function of the velocity $v_{\mathrm{D}}$ in Fig. 6 . We find only an upper limit on the velocity from the fit (seventh column in Table 2). At the $90 \%$ upper limit, we estimate that $v_{\mathrm{D}}<550 \mathrm{~km} \mathrm{~s}^{-1}$ and a thermal energy $<3.82 \mathrm{keV}$. The lower limit on the distance from the NS is $r_{\mathrm{O}}>3.5 \times 10^{9} \mathrm{~cm}$. In this case, taking into account that the geometrical thickness of the absorber is $\Delta r=\left(1.7_{-0.8}^{+0.7}\right) \times 10^{9} \mathrm{~cm}$ for a distance from the NS of $r=(1.9 \pm 0.7) \times 10^{9} \mathrm{~cm}$, we cannot exclude the possibility either that the $\mathrm{O}$ VIII absorption line forms in the outer layers of 


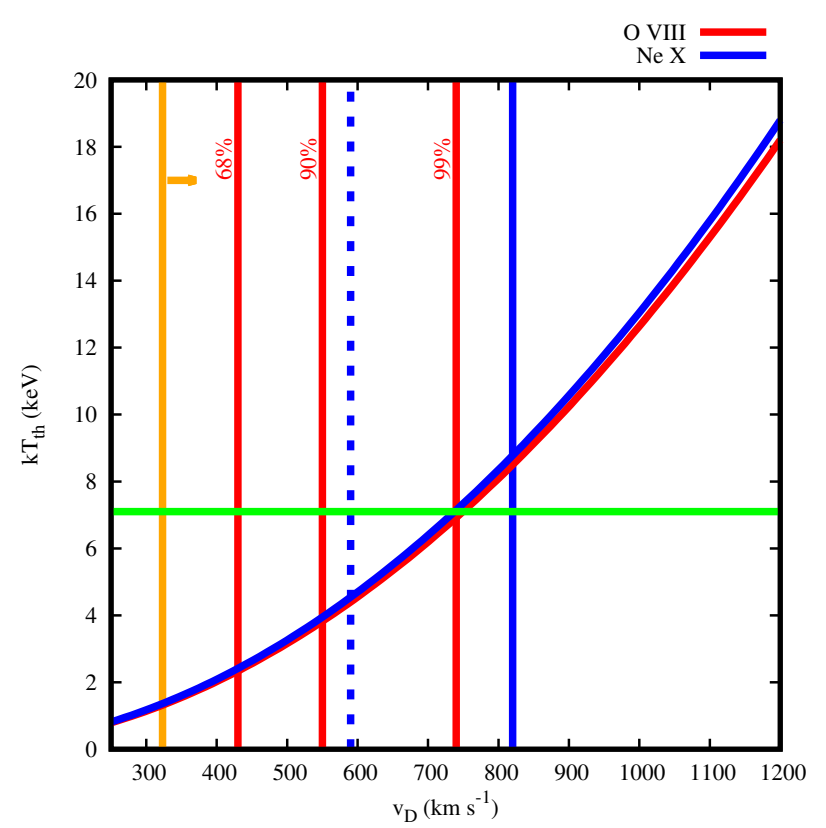

Fig. 6. Thermal temperature of the ionized absorber as a function of the velocity $v_{\mathrm{D}}$ associated with O VIII (red curve) and Ne X (blue curve). The green horizontal line indicates the thermal temperature of $7.1 \mathrm{keV}$, the solid and dashed blue vertical lines indicate the measured velocity and the corresponding uncertainties at $68 \%$ c.l. associated with the $\mathrm{Ne} \mathrm{X}$ absorption line. The red vertical lines describe the upper limit on the velocity associated with the O VIII ions at $68 \%, 90 \%$, and $99 \%$ c.l., respectively. The orange vertical line indicates the velocity $v_{\mathrm{D}}$ associated with the $\mathrm{O}$ VIII ions at the outer radius of the accretion disc.

the absorber where the other lines form. For $r_{\mathrm{O}}=3.5 \times 10^{9} \mathrm{~cm}$, we find that the fraction of $\mathrm{O}$ VIII ions with respect the whole population of oxygen atoms is $f=\left(2.2_{-0.7}^{+1.8}\right) \times 10^{-4}$, which gives an equivalent column density associated with $\mathrm{O}$ VIII ions of $6 \times 10^{16} \mathrm{~cm}^{-2}$.

Finally, we give an upper limit on the distance, $r$, from the NS of the region where the O VIII absorption line forms using the relation $n_{\mathrm{e}}=L_{\mathrm{X}} /\left(\xi r^{2}\right)$ and $N_{\mathrm{H}}=\Delta r / n_{\mathrm{e}}$. We find that $\Delta r \simeq$ $4.4 \times 10^{-10} r^{2} \mathrm{~cm}$. Knowing that the outer radius of the accretion disk is $r_{\text {disk }}=5 \times 10^{10} \mathrm{~cm}$ (Sidoli et al. 2001; Iaria et al. 2018) and imposing that the thickness of the absorber is smaller than the radial dimension of the disk, $\Delta r<r_{\text {disk }}$, we obtain that $r \lesssim$ $1.0 \times 10^{10} \mathrm{~cm}$. At this radius the turbulent velocity is $298 \mathrm{~km} \mathrm{~s}^{-1}$, the thermal velocity is $126 \mathrm{~km} \mathrm{~s}^{-1}$, the velocity $v_{\mathrm{D}} \simeq 323 \mathrm{~km} \mathrm{~s}^{-1}$, and the kinetic energy is $6.23 \mathrm{keV}$ for the oxygen ions. For $r=$ $1.0 \times 10^{10} \mathrm{~cm}$ we find that the fraction of $\mathrm{O}$ VIII ions with respect to the whole population of oxygen atoms is $f=\left(6_{-2}^{+6}\right) \times 10^{-4}$, which gives an equivalent column density associated with $\mathrm{O}$ VIII ions of $1.7 \times 10^{17} \mathrm{~cm}^{-2}$.

The physical parameters describing the ionized absorber in the soft state of MXB 1659-298 are similar to those observed in the transient eclipsing source AX J1745.6-2901 by Ponti et al. (2015), who found a column density of neutral hydrogen associated with the absorber higher than $10^{23} \mathrm{~cm}^{-2}$ and a turbulent velocity higher than $500 \mathrm{~km} \mathrm{~s}^{-1}$.

We note that the observed absorption lines we analyzed in the soft spectral state are produced within a distance of $4 \times 10^{9} \mathrm{~cm}$ from the NS; our result is compatible with the distance from the NS of the ionized absorber $\left(r \leq 1.3 \times 10^{10} \mathrm{~cm}\right)$ reported by Ponti et al. (2019). Finally, we find that the presence of an ionized absorber in the hard spectral states is also statistically significant, unlike the results by Sharma et al. (2018); this is probably related to the different continuum modeling adopted to fit the broadband spectrum.

What is true for the hard state of AX J1745.6-2901 also holds for the hard state of MXB 1659-298: the ionized iron absorption lines are less prominent, and an accurate study is not possible. The different value of the ionization parameter observed for the XMM-Newton and NuSTAR $\left(\log (\xi)_{I A}=1.98 \pm\right.$ 0.05 and $3.1 \pm 0.3$, respectively) seems to suggest a rapid ionization of the matter of the absorber, because the XMM-Newton observation was performed on 2015 September 26 and the NuSTAR observation only two days later. Finally, we observe that the broadband spectrum of MXB 1659-298 is similar to that of AX J1745.6-2901 (Ponti et al. 2015): the spectra in the soft state both show some features, such as the broad emission line in the soft state or the Compton hump in the hard state, that can be modeled by a relativistic reflection component from the accretion disk. Both spectra also show absorption lines that can be explained by an ionized absorber. A similar scenario was also adopted by Iaria et al. (2007) to describe the $\mathrm{Fe}-\mathrm{K}$ region of the dipping source X 1624-490, which has an inclination angle between $60^{\circ}$ and $70^{\circ}$. Using Chandra/HETGS data, the authors observed a broad emission line at $6.64 \mathrm{keV}$ and two narrow absorption lines associated with Fe XXV and Fe XXVI ions. Finally, the XMM-Newton spectrum of the dipping source GX $13+1$ showed evidence of a relativistically smeared reflection component with a broad emission line in the $\mathrm{Fe}-\mathrm{K}$ region and narrow absorption lines associated with highly ionized iron (see Díaz Trigo et al. 2012; Pintore et al. 2014).

Both MXB 1659-298 and AX J1745.6-2901 are binary systems with high inclination angles, and both sources show eclipses in their light curves. These similarities suggest that the broad iron lines observed in the spectra of high-inclination sources can be explained as relativistically smeared lines instead of purely Compton broadened lines. The similarities also seem to suggest a similar origin of the broad iron emission line independently of the inclination angle of the binary system.

\section{Conclusions}

We have analyzed the soft and high state of the transient eclipsing source MXB 1659-298 using XMM-Newton, NuSTAR, and Swift/XRT spectra taken during the 1999 and 2015 outburst. We find that the soft-state continuum emission can be described by a multicolor disk blackbody plus a Comptonized component. The electron temperature of the Comptonized cloud is between 2 and $4 \mathrm{keV}$ and the seed-photon temperature is between 0.4 and $0.6 \mathrm{keV}$. The inner temperature of the accretion disk is close to $0.3 \mathrm{keV}$. It is necessary to take into account a smeared reflection component to model the $\mathrm{Fe}-\mathrm{K}$ region of the spectrum where a broad emission line is observed. We find that the width of the emission line cannot be explained considering the Compton scattering alone, but we have to include the relativistic smearing in order to obtain a good fit. The reflecting region of the accretion disk is between 30 and 2800 gravitational radii, the relative reflection normalization is $0.22_{-0.05}^{+0.12}$, and the ionization parameter is $\log (\xi)=2.80_{-0.10}^{+0.20}$. Furthermore, an ionized absorber is observed with an equivalent hydrogen column density of $6 \times 10^{23} \mathrm{~cm}^{-2}$ and an ionization parameter $\log (\xi)_{\mathrm{IA}}$ between 4.1 and 4.4. Studying the narrow absorption lines associated with Fe XXVI, Fe XXV, NeX, and O VIII, we obtained information on the absorber. We find that the absorption lines associated with highly ionized iron originate at a distance from the NS of $(1.9 \pm 0.7) \times 10^{9} \mathrm{~cm}$, the one associated with $\mathrm{Ne} X$ ions originates between $(1.9 \pm 0.7) \times 10^{9} \mathrm{~cm}$ and $(3.0 \pm 1.1) \times 10^{9} \mathrm{~cm}$, and the 
line associated with $\mathrm{O}$ VIII ions originates at distances larger than $3.5 \times 10^{9} \mathrm{~cm}$.

In the hard state we do not significantly detect a thermal component associated with the direct emission of the accretion disk. The continuum emission was modeled by a Comptonized component plus a smeared reflection component. We observe an electron temperature higher than $150 \mathrm{keV}$ and a seed-photon temperature lower than $0.1 \mathrm{keV}$. The reflecting region of the accretion disk is between 6 and 290 gravitational radii, the relative reflection normalization is $0.48 \pm 0.06$, and the ionization parameter is $\log (\xi)=1.99_{-0.10}^{+0.05}$. In this state we also observed an ionized absorber with an equivalent hydrogen column density of $1.4 \times 10^{23} \mathrm{~cm}^{-2}$ and an ionization parameter $\log (\xi)_{\text {IA }}$ between 2 and 3 .

Acknowledgements. This research has made use of data and/or software provided by the High Energy Astrophysics Science Archive Research Center (HEASARC), which is a service of the Astrophysics Science Division at NASA/GSFC and the High Energy Astrophysics Division of the Smithsonian Astrophysical Observatory. The authors acknowledge financial contribution from the agreement ASI-INAF n. 2017-14-H.0, from INAF mainstream (PI: T. Belloni) and from the HERMES project financed by the Italian Space Agency (ASI) Agreement n. 2016/13 U.O. RI and TDS acknowledge the research grant iPeska (PI: Andrea Possenti) funded under the INAF national call PrinSKA/CTA approved with the Presidential Decree 70/2016. AM acknowledges funding from FSE (Fondo Sociale Europeo) Sicilia 2020

\section{References}

Burrows, D. N., Hill, J. E., Nousek, J. A., et al. 2005, Space Sci. Rev., 120, 165 Cackett, E. M., Miller, J. M., Ballantyne, D. R., et al. 2010, ApJ, 720, 205 de Vries, C. P., den Herder, J. W., Kaastra, J. S., et al. 2003, A\&A, 404, 959 den Herder, J. W., Brinkman, A. C., Kahn, S. M., et al. 2001, A\&A, 365, L7 di Salvo, T., D'Aí, A., Iaria, R., et al. 2009, MNRAS, 398, 2022

Di Salvo, T., Iaria, R., Matranga, M., et al. 2015, MNRAS, 449, 2794

Díaz Trigo, M., \& Boirin, L. 2016, Astron. Nachr., 337, 368

Díaz Trigo, M., Sidoli, L., Boirin, L., \& Parmar, A. N. 2012, A\&A, 543, A50

Done, C., \& Gierliński, M. 2006, MNRAS, 367, 659

Dove, J. B., Wilms, J., Maisack, M., \& Begelman, M. C. 1997, ApJ, 487, 759

Egron, E., Di Salvo, T., Motta, S., et al. 2013, A\&A, 550, A5

Fabian, A. C., Rees, M. J., Stella, L., \& White, N. E. 1989, MNRAS, 238, 729

Galloway, D. K., Muno, M. P., Hartman, J. M., Psaltis, D., \& Chakrabarty, D. 2008, ApJS, 179, 360

Gambino, A. F., Iaria, R., Di Salvo, T., et al. 2019, A\&A, 625, A92
Gehrels, N., Chincarini, G., Giommi, P., et al. 2004, ApJ, 611, 1005 Harrison, F. A., Craig, W. W., Christensen, F. E., et al. 2013, ApJ, 770, 103 Iaria, R., Lavagetto, G., D’Aí, A., di Salvo, T., \& Robba, N. R. 2007, A\&A, 463, 289

Iaria, R., D’Aí, A., di Salvo, T., et al. 2009, A\&A, 505, 1143

Iaria, R., Di Salvo, T., Del Santo, M., et al. 2016, A\&A, 596, A21

Iaria, R., Gambino, A. F., Di Salvo, T., et al. 2018, MNRAS, 473, 3490

Jain, C., Paul, B., Sharma, R., Jaleel, A., \& Dutta, A. 2017, MNRAS, 468, L118

Jansen, F., Lumb, D., Altieri, B., et al. 2001, A\&A, 365, L1

Kallman, T., \& Bautista, M. 2001, ApJS, 133, 221

Kallman, T. R., Palmeri, P., Bautista, M. A., Mendoza, C., \& Krolik, J. H. 2004, ApJS, 155, 675

Kolehmainen, M., Done, C., \& Díaz Trigo, M. 2011, MNRAS, 416, 311

Kotani, T., Ebisawa, K., Dotani, T., et al. 2000, ApJ, 539, 413

Mazzola, S. M., Iaria, R., Di Salvo, T., et al. 2019, A\&A, 621, A89

Miller, L., Turner, T. J., Reeves, J. N., et al. 2007, A\&A, 463, 131

Miller, J. M., Parker, M. L., Fuerst, F., et al. 2013, ApJ, 779, L2

Mitsuda, K., Inoue, H., Koyama, K., et al. 1984, PASJ, 36, 741

Muñoz-Darias, T., Fender, R. P., Motta, S. E., \& Belloni, T. M. 2014, MNRAS, 443, 3270

Özel, F., \& Freire, P. 2016, ARA\&A, 54, 401

Pandel, D., Kaaret, P., \& Corbel, S. 2008, ApJ, 688, 1288

Pintore, F., Sanna, A., Di Salvo, T., et al. 2014, MNRAS, 445, 3745

Pintore, F., Sanna, A., Di Salvo, T., et al. 2016, MNRAS, 457, 2988

Ponti, G., Bianchi, S., Muñoz-Darias, T., et al. 2015, MNRAS, 446, 1536

Ponti, G., Bianchi, S., De Marco, B., et al. 2019, MNRAS, 487, 858

Reeves, J., Done, C., Pounds, K., et al. 2008, MNRAS, 385, L108

Reis, R. C., Fabian, A. C., \& Young, A. J. 2009, MNRAS, 399, L

Risaliti, G., Bianchi, S., Matt, G., et al. 2005, ApJ, 630, L129

Sanna, A., Hiemstra, B., Méndez, M., et al. 2013, MNRAS, 432, 1144

Sanna, A., Pintore, F., Bozzo, E., et al. 2017, MNRAS, 466, 2910

Shaposhnikov, N., Titarchuk, L., \& Laurent, P. 2009, ApJ, 699, 1223

Sharma, R., Jaleel, A., Jain, C., et al. 2018, MNRAS, 481, 5560

Shimura, T., \& Takahara, F. 1995, ApJ, 445, 780

Sidoli, L., Oosterbroek, T., Parmar, A. N., Lumb, D., \& Erd, C. 2001, A\&A, 379, 540

Strüder, L., Briel, U., Dennerl, K., et al. 2001, A\&A, 365, L18

Verner, D. A., Ferland, G. J., Korista, K. T., \& Yakovlev, D. G. 1996a, ApJ, 465, 487

Verner, D. A., Verner, E. M., \& Ferland, G. J. 1996b, At. Data Nucl. Data Tables, 64,1

Wilms, J., Allen, A., \& McCray, R. 2000, ApJ, 542, 914

Woods, D. T., Klein, R. I., Castor, J. I., McKee, C. F., \& Bell, J. B. 1996, ApJ, 461,767

Yamaoka, K., Ueda, Y., Inoue, H., et al. 2001, PASJ, 53, 179

Zdziarski, A. A., Johnson, W. N., \& Magdziarz, P. 1996, MNRAS, 283, 193

Życki, P. T., Done, C., \& Smith, D. A. 1999, MNRAS, 309, 561 